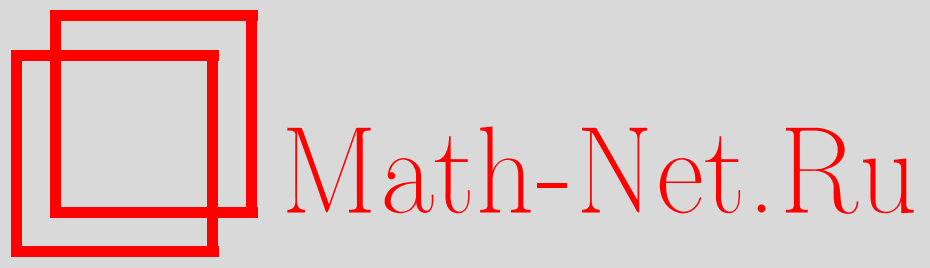

А. С. Черный, Нахождение справедливых цен на основе когерентных мер риска, Теория вероятн. и ее примен., 2007, том 52, выпуск 3, 506-540

DOI: https://doi.org/10.4213/tvp76

Использование Общероссийского математического портала Math-Net.Ru подразумевает, что вы прочитали и согласны с пользовательским соглашением

http://www . mathnet.ru/rus/agreement

Параметры загрузки:

IP : 3.80 .253 .173

26 апреля 2023 г., $17: 41: 43$

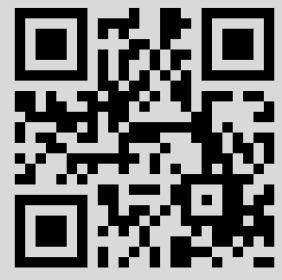




\section{НАХОЖДЕНИЕ СПРАВЕДЛИВЫХ ЦЕН НА ОСНОВЕ КОГЕРЕНТНЫХ МЕР РИСКА ${ }^{1)}$}

Это первая из двух статей, посвященных применениям когерентных мер риска к основным задачам финансовой математики. В настоящей статье рассматриваются применения этих мер к нахождению справедливых цен на неполных рынках. Доказывается фундаментальная теорема теории расчетов для метода, основанного на когерентных мерах риска. Рассматриваемая модель включает статические и динамические модели, модели с бесконечным числом активов и модели с операционными издержками. В частности, мы доказываем, что при стремлении коэффициента пропорциональных операционных издержек к нулю интервалы справедливых цен сходятся к интервалу справедливых цен в модели без издержек.

Кроме этого, мы исследуем некоторые проблемы, относящиеся собственно к мерам риска. В частности, вводится понятие генератора, открывающее путь для геометрических конструкций. С его помощью дается простое геометрическое решение проблемы размещения капитала.

Ключевые слова и фразы: взвешенный V@R, генератор, когерентная мера риска, метод NGD, операционные издержки, опорная функция, размещение капитала, риск-вклад, риск-нейтральная мера, хвостовой V@R, экстремальная мера.

\section{1. Введение}

1.1. Общий обзор. Тремя основными задачами финансовой математики можно назвать

- оптимальное инвестирование;

- нахождение справедливых цен и хеджирование;

- измерение риска и управление им.

Наиболее известные финансовые теории, относящиеся к первой задаче, - средне-квадратическая оптимизация Марковица и теория

** Московский государственный университет им. М.В. Ломоносова, механикоматематический факультет, кафедра теории вероятностей, Ленинские горы, 119992 Москва, Россия; e-mail: alexander.cherny@gmail.com; страница в интернете: http://mech.math.msu.su/ cherny. 
САРМ Шарпа, которые часто называют «первой революцией в финансовой математике». Наиболее известный результат, относящийся ко второй задаче, - формула Блэка-Шоулса-Мертона, которую часто называют «второй революцией в финансовой математике». Недавно было введено новое фундаментальное понятие, относящееся к третьей задаче. В 1997 г. Артцнер, Делбаен, Эбер и Хис [4], [5] ввели понятие когерентной меры риска как нового способа измерения риска. Начиная с этих работ, теория когерентных мер риска очень быстро развивается, и уже сейчас в некоторых источниках ее называют «третьей революцией в финансовой математике» (см. [68]). Упомянем, в частности, работы [1], $[3],[21],[34],[35],[43],[49],[69]$ и обзоры [22], [36, гл. 4], [60]. В настоящее время одной из главных задач этой теории является определение когерентных мер риска в динамической постановке; см., в частности, работы [12], [27], [41], [55] и [57].

Теория когерентных мер важна не только для задачи измерения риска. Действительно, риск ( $\approx$ неопределенность) лежит в основе всей теории финансов, и поэтому новый подход к его измерению приводит к новым подходам к другим задачам теории финансов, в частности, относящимся к первой и второй задачам. Сейчас все больше и больше работ направлены именно на применения когерентных мер к различным финансовым проблемам. На самом деле, всю финансовую математику можно построить на когерентных мерах риска.

Одна из главных проблем, стоящих перед современной финансовой математикой, - нахождение как можно более узких границ для цен производных инструментов в неполных моделях. Как известно, интервалы цен, которые дает теория арбитража, неприемлемо широки в большинстве неполных моделей. Для их сужения требуются принципиально новые идеи. Недавно появился многообещающий подход, названный методом отсутствия выъодных операчий; см. [6], [17]. Проиллюстрируем его идею на примере. Рассмотрим контракт, дающий его владельцу сумму 0 с вероятностью $\frac{1}{2}$ и сумму 100 рублей с вероятностью $\frac{1}{2}$. Тогда безарбитражным интервалом цен для этого контракта служит интервал $(0,100)$. В то же время ясно, что если этот контракт стоит 1 рубль, то все участники рынка захотят его приобрести, так что предложение не уравновесит спрос. Итак, цена в 1 рубль нереалистична, поскольку приводит к появлению операции, выгодной для всех участников рынка (именно, покупке этого контракта). Метод отсутствия выгодных операций (в дальнейшем будем использовать аббревиатуру NGD, происходящую от английского термина No Good Deals) основан на предположении, что выгодные операции отсутствуют.

Немедленно возникает проблема: как математически определить выгодную операцию? В работах [6], [17] под выгодной операцией понимается такая, у которой отношение прибыль/риск превышает задан- 
ный порог. При этом Кохран и Саa-Рекехо [17] использовали дробь Шарпа, а Бернардо и Ледуа [6] воспользовались другим отношением прибыль/риск. Черни и Ходжез [11] предложили обобщение обоих определений (см. также работу Бьорка и Слинко [7], обобщающую результаты [17]).

По-другому метод NGD можно мотивировать следующим образом. Когда агент продает опцион, он назначает цену, при помощи которой он может суперхеджировать этот опцион. В теории суперхеджирование обычно понимается почти наверное, но на практике агент старается достичь меньшего, а именно, чтобы риск его позиции оставался в заданных пределах (поскольку суперхеджирование почти наверное на практике обычно невозможно). Эти рассмотрения приводят к методу NGD, в котором под выгодной операцией понимается операция с отрицательным риском (такое понимание альтернативно работам [6], [7], [11], [17], в которых под выгодной операцией понимается операция с положительным риском, но большим отношением прибыль/риск), а под хеджированием понимается минимизация риска.

Tрадиционные способы измерения риска - дисперсия и V@R (Value at Risk) ${ }^{2)}$. Если риск измерять при помощи дисперсии, то его минимизация есть метод средне-квадратического хеджирования, введенный Даффи и Ричардсоном [29] и развитый в работах [53], [61], [62] (см. также [36, гл. 10]). Если риск измерять при помощи V@R, то его минимизация есть метод квантильного хеджирования, введенный Фёльмером и Лейкертом [33] (см. также [36, гл. 8]).

Но более правильно для измерения риска использовать не дисперсию и $\mathrm{V} @ \mathrm{R}$, а когерентные меры риска. Соответствующий метод нахождения цены был изучен в ряде статей. Карр, Жеман и Мадан [9] (см. также обзор [10]) рассмотрели его в вероятностной постановке (хотя в их работе и не появляется термин «выгодная операция»), а Яшке и Кюхлер [40] исследовали его в постановке топологических пространств в стиле Харрисона и Крепса [37] (см. также работу Стаума [66], обобщающую [40]). Упомянем также работы [41], [50] и [57], в которых рассматривается метод NGD, основанный на динамических когерентных мерах риска и на выпуклых мерах риска.

1.2. Цель работы. Это первая из цикла работ, посвященных применениям когерентных мер риска к основным задачам финансовой математики (другая работа в серии - статья [15]). В настоящей статье мы рассматриваем применения этих мер риска к нахождению справед-

2) Напомним, что для портфеля, приносящего случайную прибыль $X$ (под прибылью здесь понимается разность между конечной и начальной стоимостью портфеля), Value at Risk определяется как $\mathrm{V} @ \mathrm{R}_{\lambda}(X)=-q_{\lambda}(X)$, где $q_{\lambda}$ обозначает $\lambda$-квантиль, а $\lambda \in(0,1)$ - фиксированный параметр (обычно берется $\lambda=0.05$ или $\lambda=0.01$ ). 
ливых цен в неполных моделях. Изучается метод NGD в двух формах: в первом случае под выгодной операцией понимается операция с отрицательным риском, а во втором - операция с положительным риском, но высоким отношением прибыль/риск (при этом оказывается, что второй метод сводится к первому заменой меры риска). Значительный пласт исследований в финансовой математике связан с фундаментальной теоремой теории расчетов для метода отсутствия арбитража (достаточно упомянуть работы [14], [20], [23], [24], [37]-[39], [44], [46], [56], [58], [67], [70]; см. также [64, гл. 5, 7]). Мы доказываем эту теорему для метода NGD (теорема 3.1). Полученная теорема является весьма общей ${ }^{3)}$ : она применима к произвольным вероятностным пространствам, широкому классу когерентных мер риска (включающему все естественные когерентные меры риска), моделям непрерывного времени, моделям с бесконечным числом активов и моделям с операционными издержками ${ }^{4)}$. Как следствие из доказанной теоремы, мы получаем вид интервалов справедливых цен (следствие 3.1).

Недавно внимание ряда исследователей привлекла следующая проблема. Рассмотрим модель с пропорциональными операционными издержками. Верно ли, что при стремлении коэффициента операционных издержек к нулю верхняя (соответственно нижняя) цена платежного поручения в этой модели сходится к верхней (соответственно нижней) цене этого же поручения в модели без издержек? В работах [14], [19], [51] и [65] было показано, что для метода отсутствия арбитража ответ на этот вопрос отрицателен уже в модели Блэка-Шоулса-Мертона (в качестве платежного поручения рассматривался опцион колл европейского типа). Этот результат можно интерпретировать так: метод отсутствия арбитража бесполезен в моделях непрерывного времени с операционными издержками. В этой работе мы доказываем, что при использовании метода NGD ответ на этот вопрос положителен (теорема 3.2). Это доказывается в рамках общей модели с бесконечным числом активов и для произвольного платежного поручения (при этом не делается никаких предположений о вероятностной структуре процесса цены). Достоинством метода NGD служит не только то, что этот результат верен в такой общей постановке, но и то, что он допускает короткое доказательство.

3) Можно сказать, что наш подход обобщает [9], но это является обобщением сразу во многих направлениях: в работе [9] используются (нереалистичные) предположения о конечности вероятностного пространства и конечности множества вероятностных сценариев, определяющих меру риска (все естественные когерентные меры риска не удовлетворяют этому условию; см. п. 2.1). Кроме того, в [9] рассматривается лишь статическая модель с конечным числом активов, причем без операционных издержек. Также в [9] не рассматривались отношения прибыль/риск.

4) «Безарбитражной» фундаментальной теореме для моделей с операционными издержками посвящены, например, работы [14], [25], [42], [45], [47], [59]. 
Хотя этот цикл работ нацелен прежде всего на применения когерентных мер риска к задачам финансовой математики, мы также устанавливаем ряд результатов и даем определения, относящиеся собственно к теории мер риска. В частности, мы вводим понятие экстремальной мерьл. Результаты этой статьи и статьи [15] показывают важность и удобство этого понятия: через него выражаются справедливые цены в ряде методов, предложенных в [15]; оно также появляется в связи с равновесием в [15]. В настоящей работе мы даем решение проблемы размещения капитала в терминах экстремальных мер (теорема 2.2). Отметим, что эта задача рассматривалась в работах [22], [26], [31], [48], [54] и [69].

Наряду с измерением собственно риска, очень важна задача измерения риск-вклада подпортфеля в большой портфель. Основываясь на полученном решении задачи размещения капитала, мы даем несколько эквивалентных определений когерентного риск-вклада.

Также в статье вводится понятие генератора. Оно устанавливает связь между когерентными рисками и выпуклым анализом, открывая путь для применений геометрии. В частности, дается (рис. 1) геометрическое решение задачи размещения капитала (таким образом, приводятся два решения: вероятностное дается в терминах экстремальных мер, а геометрическое - в терминах генераторов). Кроме того, предлагается геометрическое решение задачи NGD-суперхеджирования (см. рис. 4). В работе [15] мы приводим геометрические решения задач оптимизации, оптимизационного нахождения цены и равновесия. Для большинства рассмотренных задач в настоящей работе и в [15] предлагаются два вида результатов:

- вероятностный результат, применимый к общей модели, дается во многих случаях в терминах экстремальных мер;

- геометрический результат, применимый к статической модели с конечным числом активов, дается в терминах генераторов.

Подводя итог, отметим ряд преимуществ метода NGD, основанного на когерентных мерах риска, в сравнении с такими методами, как отсутствие арбитража, средне-квадратическое хеджирование, квантильное хеджирование и методы NGD, основанные на других отношениях прибыль/риск.

- Получаемые интервалы справедливых цен являются более узкими, чем безарбитражные интервалы. В частности, имеет место сходимость интервалов в моделях с операционными издержками.

- Метод является гибким в том смысле, что его можно применять с разными мерами риска. В то же время другие методы не обладают такой гибкостью.

- Метод допускает как модификацию, где под выгодной операцией понимается операция с отрицательным риском, так и модификацию, где под выгодной операцией понимается операция с высоким отношением 
прибыль/риск, причем вторая сводится к первой простой заменой меры риска. Остальные же методы не обладают таким свойством инвариантности.

- Поскольку когерентные меры риска определяются через семейство вероятностных мер, то возникает богатая теория двойственности.

- Введение понятия генератора устанавливает связь с геометрией и открывает путь для применений выпуклого анализа.

1.3. Структура работы. Раздел 2 посвящен собственно когерентным мерам риска. В п. 2.1 мы напоминаем основные определения и факты. В п. 2.2 вводятся $L^{1}$-пространства, связанные с когерентными мерами риска (они используются в технических условиях в дальнейших теоремах). Пункт 2.3 содержит определение экстремальной меры. В п. 2.4 дается решение проблемы размещения капитала. В п. 2.5 предлагаются эквивалентные определения когерентного риск-вклада.

Раздел 3 посвящен нахождению цены при помощи метода NGD. В пп. 3.1 и 3.2 рассматриваются две формы метода NGD в рамках общей модели. В пп. 3.3-3.5 рассматриваются применения к более конкретным моделям. Далее, в п. 3.6 приводится геометрическое решение проблемы NGD-суперхеджирования для статической модели с конечным числом активов.

\section{2. Когерентные меры риска}

2.1. Основные определения. Пусть $(\Omega, \mathscr{F}, \mathbf{P})$ - вероятностное пространство. Следующее определение было введено в работах [4], [5]. Точнее, там определялись когерентные меры риска на конечном $\Omega$; в этом случае аксиома (е) (см. ниже) не нужна. Она была введена Делбаеном [21] для произвольного $\Omega$. В теории удобнее иметь дело не с когерентными мерами риска, а с противоположными им по знаку величинами - когерентными функциями полезности (это понятие было введено в [22]). Это позволяет избежать большого количества минусов.

О п р е д е л е н и 2.1. Когерентная функиия полезности на $L^{\infty}$ - это отображение $u: L^{\infty} \rightarrow \mathbf{R}$ со свойствами:

(a) (супераддитивность) $u(X+Y) \geqslant u(X)+u(Y)$;

(b) (монотонность) если $X \leqslant Y$, то $u(X) \leqslant u(Y)$;

(c) (положительная однородность) $u(\lambda X)=\lambda u(X)$ для $\lambda \in \mathbf{R}_{+}$;

(d) (инвариантность относительно сдвигов) $u(X+m)=u(X)+m$ для $m \in \mathbf{R}$;

(е) (свойство Фату) если $\left|X_{n}\right| \leqslant 1, X_{n} \stackrel{\mathbf{P}}{\longrightarrow} X$, то

$$
u(X) \geqslant \limsup _{n} u\left(X_{n}\right) .
$$

Соответствующая когерентная мера риска определяется как $\rho(X)=-u(X)$. 
3 а м е ч а н и е. Обычно когерентная мера риска определяется через условия (a)-(d), а потом рассматриваются меры риска со свойством Фату. Однако лишь такие меры риска полезны, и поэтому нам удобнее сразу включить свойство (е) в список базовых аксиом.

Теорема, приведенная ниже, была получена в [5] для конечного $\Omega$ (в этом случае аксиома (е) не нужна) и в [21] для общего случая. Будем обозначать через $\mathscr{P}$ множество вероятностных мер, абсолютно непрерывных относительно Р. В данной статье мы отождествляем меры из $\mathscr{P}$ (они обычно обозначаются через $\mathbf{Q}$ ) с их плотностями относительно $\mathbf{P}$ (они обычно обозначаются через $Z$ ).

Теорема 2.1 (основная теорема о представлении). Отображение и удовлетворяет условиям (а)-(е) тогда и только тогда, когда существует непустое множество $\mathscr{D} \subseteq \mathscr{P}$ такое, ито

$$
u(X)=\inf _{\mathbf{Q} \in \mathscr{D}} \mathbf{E}_{\mathbf{Q}} X, \quad X \in L^{\infty}
$$

3 а м е ч а н и е. Меры из множества $\mathscr{D}$ иногда называют вероятностными сценариями, задающими меру риска $\rho$.

До сих пор когерентные меры риска определялись на ограниченных случайных величинах. В то же время почти все распределения, используемые в финансовой математике, неограничены (как, например, гауссовское или логнормальное). Представляется безнадежным пытаться аксиоматизировать меры риска на пространстве $L^{0}$ всех случайных величин и потом получать теорему о представлении. Вместо этого мы берем представление (2.1) за основу и переносим его на $L^{0}$.

О п р е д е л н и е 2.2. Когерентная функиия полезности на $L^{0}$ - это отображение $u: L^{0} \rightarrow[-\infty, \infty]$ вида

$$
u(X)=\inf _{\mathbf{Q} \in \mathscr{D}} \mathbf{E}_{\mathbf{Q}} X, \quad X \in L^{0},
$$

где $\mathscr{D} \subseteq \mathscr{P}$, а $\mathbf{E}_{\mathbf{Q}} X$ понимается как $\mathbf{E}_{\mathbf{Q}} X^{+}-\mathbf{E}_{\mathbf{Q}} X^{-}$с соглашением $\infty-\infty=-\infty$. Соответствующая когерентная мера риска определяется как $\rho(X)=-u(X)$.

Очевидно, множество $\mathscr{D}$, для которого имеет место представление (2.1) или (2.2), не единственно. Однако существует наибольшее такое множество. Оно имеет вид $\left\{\mathbf{Q} \in \mathscr{P}: \mathbf{E}_{\mathbf{Q}} X \geqslant u(X)\right.$ для любого $\left.X\right\}$. Введем определение.

О п р е д е л е н и е 2.3. Пусть $u$ - когерентная функция полезности на $L^{\infty}$ (соответственно $\left.L^{0}\right)$. Назовем наибольшее множество, для которого выполнено (2.1) (соответственно (2.2)), определяющим множеством для $u$. 
З а м е ч а н и е. Очевидно, определяющее множество выпукло. Для когерентных функций полезности на $L^{\infty}$ оно также $L^{1}$-замкнуто. Однако для когерентных функций полезности на $L^{0}$ оно может не быть $L^{1}$-замкнутым. В качестве примера возьмем положительную неограниченную случайную величину $X_{0}$ такую, что $\mathbf{P}\left\{X_{0}=0\right\}>0$, и рассмотрим $\mathscr{D}_{0}=\left\{\mathbf{Q} \in \mathscr{P}: \mathbf{E}_{\mathbf{Q}} X_{0}=1\right\}$. Очевидно, определяющее множество $\mathscr{D}$ когерентной функции полезности $u(X)=\inf \mathbf{Q}_{\mathbf{Q} \in \mathscr{D}_{0}} \mathbf{E}_{\mathbf{Q}} X$ удовлетворяет условию $\mathscr{D}_{0} \subseteq \mathscr{D} \subseteq\left\{\mathbf{Q} \in \mathscr{P}: \mathbf{E}_{\mathbf{Q}} X_{0} \geqslant 1\right\}$. С другой стороны, $L^{1}$-замыкание $\mathscr{D}_{0}$ содержит меры, сосредоточенные на $\left\{X_{0}=0\right\}$.

В а ж н о е 3 а м е ч а н и е. Пусть $\mathscr{D}-L^{1}$-выпуклое замкнутое подмножество $\mathscr{P}$. (На самом деле наибольшую важность представляет случай, когда $\mathscr{D} L^{1}$-замкнуто, выпукло и равномерно интегрируемо; это предположение будет нужно в ряде условий ниже.) Определим когерентную функцию полезности $u$ по формуле (2.2). Тогда $\mathscr{D}$ служит определяющим множеством для $u$. Действительно, допустим, что определяющее множество $\widetilde{\mathscr{D}}$ больше, чем $\mathscr{D}$, т.е. существует $\mathrm{Q}_{0} \in \widetilde{\mathscr{D}} \backslash \mathscr{D}$. По теореме Хана-Банаха можно найти элемент $X_{0} \in L^{\infty}$ такой, что $\mathbf{E}_{\mathbf{Q}_{0}} X_{0}<\inf _{\mathbf{Q} \in \mathscr{D}} \mathbf{E}_{\mathbf{Q}} X$, что является противоречием. То же рассуждение показывает, что $\mathscr{D}$ также служит определяющим множеством для сужения $u$ на $L^{\infty}$.

В дальнейшем будем всегда рассматривать когерентные функции полезности на $L^{0}$.

П р и м е р 2.1. (i) Хвостовой $V @ R$ (в англоязычной литературе используются термины Tail V@R, Average V@R, Conditional V@R и Expected Shortfall) - это мера риска, отвечающая когерентной функции полезности

$$
u_{\lambda}(X)=\inf _{\mathbf{Q} \in \mathscr{D}_{\lambda}} \mathbf{E}_{\mathbf{Q}} X
$$

где $\lambda \in(0,1]$ и

$$
\mathscr{D}_{\lambda}=\left\{\mathbf{Q} \in \mathscr{P}: \frac{d \mathbf{Q}}{d \mathbf{P}} \leqslant \lambda^{-1}\right\} .
$$

Предположим, что $X$ имеет непрерывное распределение и положим $Z_{*}=$ $\lambda^{-1} I\left(X \leqslant q_{\lambda}\right)$, где $q_{\lambda}-\lambda$-квантиль распределения $X$. Тогда

$$
\begin{aligned}
\left(X-q_{\lambda}\right) Z-\left(X-q_{\lambda}\right) Z_{*}= & \left(X-q_{\lambda}\right)\left(Z-\lambda^{-1}\right) I\left(X-q_{\lambda}<0\right) \\
& +\left(X-q_{\lambda}\right) Z I\left(X-q_{\lambda}>0\right) \geqslant 0
\end{aligned}
$$

для любого $Z \in \mathscr{D}_{\lambda}$, откуда

$$
u_{\lambda}(X)=\mathbf{E} Z_{*} X=\mathbf{E}\left(X \mid X \leqslant q_{\lambda}\right)
$$

(это оправдывает термин хвостовой $\mathrm{V} @ \mathrm{R}$ ). Подробное изучение этой меры риска содержится в [3], [21, раздел 6], [22, раздел 7], [36, раздел 4.4], [60, раздел 1.3]. 
(ii) Bзвешенный $V @ R$ на $L^{\infty}$ (в англоязычной литературе используются термины Weighted V@R и Spectral risk measure) - это мера риска, отвечающая когерентной функции полезности

$$
u_{\mu}(X)=\int_{(0,1]} u_{\lambda}(X) \mu(d \lambda), \quad X \in L^{\infty},
$$

где $\mu$ - вероятностная мера на $(0,1]$.

Взвешенный $V @ R$ на $L^{0}$ определяется как мера риска, соответствующая когерентной функции полезности

$$
u_{\mu}(X)=\inf _{\mathbf{Q} \in \mathscr{D}_{\mu}} \mathbf{E}_{\mathbf{Q}} X, \quad X \in L^{0},
$$

где $\mathscr{D}_{\mu}$ - определяющее множество для $u_{\mu}$ на $L^{\infty}$ (при этом равенство (2.5) сохраняется на $L^{0}$; см. [16, теорема 3.2$\left.]\right)$.

Отметим, что при некоторых условиях регулярности на меру $\mu$ взвешенный V@R обладает рядом важных свойств, которыми не обладает хвостовой V@R. Взвешенный V@R является более «гладкой» мерой риска, чем хвостовой V@R. C нашей точки зрения, взвешенный V@R один из важнейших классов когерентных мер риска. Детальное изучение этой меры риска содержится в работах [1], [2], [28], [49], а также в статье [16], являющейся в некотором смысле продолжением настоящей работы и статьи [15].

2.2. Пространства $L_{\mathrm{w}}^{1}$ и $L_{\mathrm{s}}^{1}$. Для множества $\mathscr{D} \subseteq \mathscr{P}$ введем слабое и сильное $L^{1}$-пространства:

$$
\begin{aligned}
L_{\mathrm{w}}^{1}(\mathscr{D}) & =\left\{X \in L^{0}: u(X)>-\infty, u(-X)>-\infty\right\} \\
L_{\mathrm{s}}^{1}(\mathscr{D}) & =\left\{X \in L^{0}: \lim _{n \rightarrow \infty} \sup _{\mathbf{Q} \in \mathscr{D}} \mathbf{E}_{\mathbf{Q}}|X| I(|X|>n)=0\right\}
\end{aligned}
$$

Очевидно, $L_{\mathbf{s}}^{1}(\mathscr{D}) \subseteq L_{\mathrm{w}}^{1}(\mathscr{D})$. Если $\mathscr{D}=\{\mathbf{Q}\}-$ одноточечное множество, то $L_{\mathrm{w}}^{1}(\mathscr{D})=L_{\mathrm{s}}^{1}(\mathscr{D})=L^{1}(\mathbf{Q})$, что оправдывает определение.

В общем случае $L_{\mathrm{s}}^{1}(\mathscr{D})$ может быть строго меньше, чем $L_{\mathrm{w}}^{1}(\mathscr{D})$. K примеру, пусть $X_{0}$ - положительная неограниченная случайная величина с $\mathbf{P}\left\{X_{0}=0\right\}>0$, и положим $\mathscr{D}=\left\{\mathbf{Q} \in \mathscr{P}: \mathbf{E}_{\mathbf{Q}} X_{0}=1\right\}$. Тогда $X_{0} \in L_{\mathrm{w}}^{1}(\mathscr{D})$, но $X_{0} \notin L_{\mathrm{s}}^{1}(\mathscr{D})$. (Можно построить аналогичный пример с $L^{1}$-замкнутым $\mathscr{D}$; см. пример 2.3.) Однако, как видно из предложения ниже, в наиболее естественных случаях сильное и слабое пространства совпадают.

Предложение 2.1. (i) Если $\mathscr{D}_{\lambda}-$ определяющее множество хвоcтового V@R (см. пример 2.1(i)), то $L_{\mathrm{w}}^{1}\left(\mathscr{D}_{\lambda}\right)=L_{\mathrm{s}}^{1}\left(\mathscr{D}_{\lambda}\right)$.

(ii) Если $\mathscr{D}_{\mu}$ - определяюиее множество взвешенного $V @ R$ (см. пример 2.1 (ii)), mо $L_{\mathrm{w}}^{1}\left(\mathscr{D}_{\mu}\right)=L_{\mathrm{s}}^{1}\left(\mathscr{D}_{\mu}\right)$. 
(iii) Если все плотности из $\mathscr{D}$ ограниченьл единой константой и $\mathbf{P} \in \mathscr{D}, \operatorname{mo} L_{\mathrm{w}}^{1}(\mathscr{D})=L_{\mathrm{s}}^{1}(\mathscr{D})$.

(iv) Если $\mathscr{D}$ является вьпуклой комбинацией $\sum_{n=1}^{N} a_{n} \mathscr{D}_{n}$, где $\mathscr{D}_{1}, \ldots, \mathscr{D}_{N}$ maкuе, ито $L_{\mathrm{w}}^{1}\left(\mathscr{D}_{n}\right)=L_{\mathrm{s}}^{1}\left(\mathscr{D}_{n}\right)$, mo $L_{\mathrm{w}}^{1}(\mathscr{D})=L_{\mathrm{s}}^{1}(\mathscr{D})$.

(v) $E c л u \mathscr{D}=\operatorname{conv}\left(\mathscr{D}_{1}, \ldots, \mathscr{D}_{N}\right)$, где $\mathscr{D}_{1}, \ldots, \mathscr{D}_{N}$ maкue, ито $L_{\mathrm{w}}^{1}\left(\mathscr{D}_{n}\right)=L_{\mathrm{s}}^{1}\left(\mathscr{D}_{n}\right)$, mo $L_{\mathrm{w}}^{1}(\mathscr{D})=L_{\mathrm{s}}^{1}(\mathscr{D})$.

Лемма 2.1. Если $\mu$ является выпуклой комбинаиией $\sum_{n=1}^{\infty} a_{n} \delta_{\lambda_{n}}$, то определяюшее множество $\mathscr{D}_{\mu}$ взвешенного V@R имеет вид $\sum_{n=1}^{\infty} a_{n} \mathscr{D}_{\lambda_{n}}$, где $\mathscr{D}_{\lambda}$ задано равенством (2.3).

Д ок а з а т е льст в о. Обозначим $\sum_{n} a_{n} \mathscr{D}_{\lambda_{n}}$ через $\mathscr{D}$. Очевидно, $\mathscr{D}$ выпукло. Фиксируем $X \in L^{\infty}$. Легко видеть, что для любого $n$ минимум математических ожиданий $\mathbf{E} X Z$ по $Z \in \mathscr{D}_{\lambda_{n}}$ достигается на случайной величине $Z_{k}=\lambda_{k}^{-1} I\left(X<q_{\lambda_{k}}\right)+c_{k} I\left(X=q_{\lambda_{k}}\right)$, где $q_{\lambda_{k}}-\lambda_{k^{-}}$ квантиль распределения $Z_{k}$, а $c_{k}$ выбрано так, что $\mathbf{E} Z_{k}=1$ (доказательство аналогично рассуждению из примера 2.1 (i)). Поэтому минимум ожиданий $\mathbf{E}_{\mathbf{p}} X Z$ по $Z \in \mathscr{D}$ также достигается. По теореме Джеймса (см. [32]) $\mathscr{D}$ слабо компактно. В частности, оно $L^{1}$-замкнуто.

Очевидно, $u_{\mu}(X)=\inf _{\mathbf{Q} \in \mathscr{D}} \mathbf{E}_{\mathbf{Q}} X$ для любого $X \in L^{\infty}$. Принимая во внимание важное замечание после определения 2.3 , получаем $\mathscr{D}_{\mu}=\mathscr{D}$. Лемма 2.1 доказана.

Доказательст в о п редл о жен ия 2.1. Единственным нетривиальным утверждением является (ii). Для его доказательства рассмотрим меры $\tilde{\mu}=\sum_{k=1}^{\infty} a_{k} \delta_{2^{-k}}, \bar{\mu}=\sum_{k=1}^{\infty} a_{k} \delta_{2^{-k+1}}$, где $a_{k}=$ $\mu\left(\left(2^{-k}, 2^{-k+1}\right]\right)$. Поскольку $u_{\widetilde{\mu}} \leqslant u_{\mu} \leqslant u_{\bar{\mu}}$, имеем $\mathscr{D}_{\widetilde{\mu}} \supseteq \mathscr{D}_{\mu} \supseteq \mathscr{D}_{\bar{\mu}}$. По лемме 2.1

$$
\mathscr{D}_{\widetilde{\mu}}=\left\{\sum_{k=1}^{\infty} a_{k} Z_{k}: Z_{k} \in \mathscr{D}_{2-k}\right\}, \quad \mathscr{D}_{\bar{\mu}}=\left\{\sum_{k=1}^{\infty} a_{k} Z_{k}: Z_{k} \in \mathscr{D}_{2^{-k+1}}\right\} .
$$

Возьмем $X \in L_{\mathrm{w}}^{1}\left(\mathscr{D}_{\mu}\right)$. Рассмотрим $Z_{k}=2^{k-1} I\left(X<q_{k}\right)+c_{k} I\left(X=q_{k}\right)$, где $q_{k}-2^{-k+1}$-квантиль распределения $X$, а $c_{k}$ выбрано так, что $\mathbf{E}_{\mathbf{P}} Z_{k}=1$. Тогда

$$
\mathbf{E}_{\mathbf{P}} Z_{k} X=\min _{Z \in \mathscr{D}_{2^{-k+1}}} \mathbf{E}_{\mathbf{P}} Z X
$$

Плотность $Z_{0}=\sum_{k=1}^{\infty} a_{k} Z_{k}$ принадлежит $\mathscr{D}_{\bar{\mu}}$ и

$$
\mathbf{E}_{\mathbf{P}} Z_{0} X=\min _{Z \in \mathscr{D}_{\bar{\mu}}} \mathbf{E}_{\mathbf{P}} Z X
$$

Ввиду включения $X \in L_{\mathrm{w}}^{1}\left(\mathscr{D}_{\mu}\right) \subseteq L_{\mathrm{w}}^{1}\left(\mathscr{D}_{\bar{\mu}}\right)$ последнее выражение конечно, т.е.

$$
\sum_{k=1}^{\infty} a_{k} \min _{Z \in \mathscr{D}_{2}-k+1} \mathbf{E}_{\mathbf{P}} Z X>-\infty
$$


откуда

$$
\sum_{k=1}^{\infty} a_{k} \min _{Z \in \mathscr{D}_{2-k+1}} \mathbf{E}_{\mathbf{P}} Z\left(-X^{-}\right)>-\infty
$$

Такая же оценка верна и для $X^{+}$, так что

$$
\sum_{k=1}^{\infty} a_{k} \sup _{Z \in \mathscr{D}_{2^{-k}}} \mathbf{E}_{\mathbf{P}} Z|X| \leqslant 2 \sum_{k=1}^{\infty} a_{k} \sup _{Z \in \mathscr{D}_{2^{-k+1}}} \mathbf{E}_{\mathbf{P}} Z|X|<\infty .
$$

Очевидно, что $X \in L^{1}$, так что для любого $k$

$$
\sup _{Z \in \mathscr{D}_{2-k}} \mathbf{E}_{\mathbf{P}} Z|X| I(|X|>n) \leqslant 2^{k} \mathbf{E}_{\mathbf{P}}|X| I(|X|>n) \underset{n \rightarrow \infty}{\longrightarrow} 0 .
$$

Принимая во внимание (2.6), получаем

$$
\begin{aligned}
\sup _{Z \in \mathscr{D}_{\mu}} \mathbf{E}_{\mathbf{P}} Z|X| I(|X|>n) & \leqslant \sup _{Z \in \mathscr{D}_{\tilde{\mu}}} \mathbf{E}_{\mathbf{P}} Z|X| I(|X|>n) \\
& =\sum_{k=1}^{\infty} a_{k} \sup _{Z \in \mathscr{D}_{2^{-k}}} \mathbf{E}_{\mathbf{P}} Z|X| I(|X|>n) \underset{n \rightarrow \infty}{\longrightarrow} 0
\end{aligned}
$$

\section{3. Экстремальные меры.}

О п р е д е л е н и е 2.4. Пусть $u$ - когерентная функция полезности с определяющим множеством $\mathscr{D}$. Пусть $X \in L^{0}$. Назовем меру $\mathbf{Q} \in \mathscr{D}$ экстремальной мерой для $X$, если $\mathbf{E}_{\mathbf{Q}} X=u(X) \in(-\infty, \infty)$. Множество экстремальных мер будем обозначать через $\mathscr{X}_{\mathscr{D}}(X)$.

Напомним некоторые общие факты о слабой топологии на $L^{1}$. Csaбая топология на $L^{1}$ индуцирована двойственностью между $L^{1}$ и $L^{\infty}$ и обычно обозначается $\sigma\left(L^{1}, L^{\infty}\right)$. Критерий Данфорда-Петтиса утверждает, что множество $\mathscr{D} \subseteq \mathscr{P}$ слабо компактно тогда и только тогда, когда оно слабо замкнуто и равномерно интегрируемо. Далее, применение теоремы Хана-Банаха показывает, что выпуклое множество $\mathscr{D} \subseteq \mathscr{P}$ слабо замкнуто тогда и только тогда, когда оно $L^{1}$-замкнуто.

Предложение 2.2. Если определяюшее множество $\mathscr{D}$ слабо замкнуто, а $X \in L_{\mathrm{s}}^{1}(\mathscr{D})$, mо $\mathscr{X}_{\mathscr{D}}(X) \neq \varnothing$.

Д ок а з а т е л ь с т в о. Очевидно, $u(X) \in(-\infty, \infty)$. Выберем последовательность $Z_{n} \in \mathscr{D}$ такую, что $\mathbf{E}_{\mathrm{P}} Z_{n} X \rightarrow u(X)$. Эта последовательность имеет слабую предельную точку $Z_{\infty} \in \mathscr{D}$. Очевидно, отображение $\mathscr{D} \ni Z \mapsto \mathbf{E}_{\mathbf{P}} Z X$ слабо непрерывно. Отсюда $\mathbf{E}_{\mathbf{P}} Z_{\infty} X=u(X)$. Это означает, что $Z_{\infty} \in \mathscr{X}_{\mathscr{D}}(X)$.

П р и м е p 2.2. (i) Если $u$ отвечает хвостовому V@R порядка $\lambda$ (см. пример $2.1(\mathrm{i})$ ), а $X$ имеет непрерывное распределение, то, как легко видеть из $(2.4), \mathscr{X}_{\mathscr{D}}(X)$ состоит из единственной плотности $\lambda^{-1} I(X \leqslant$ $\left.q_{\lambda}\right)$, где $q_{\lambda}-\lambda$-квантиль распределения $X$. 
(ii) Если $u$ отвечает взвешенному V@R c мерой $\mu$ (см. пример 2.1 (ii)), а $X$ имеет непрерывное распределение, то $\mathscr{X}_{\mathscr{D}}(X)$ состоит из единственной плотности $g(X)$, где $g(x)=\int_{[F(x), 1]} \lambda^{-1} \mu(d \lambda)$, а $F$ - функция распределения $X$ (см. [16, раздел 6]). Заметим, что эта плотность отражает неприятие риска агентом, чей капитал приносит прибыль $X$.

Условие слабой компактности $\mathscr{D}$ является очень слабым и выполнено для определяющих множеств большинства естественных мер риска. Очевидно, определяющее множество $\mathscr{D}_{\lambda}$ хвостового V@R удовлетворяет этому условию. Определяюшее множество $\mathscr{D}_{\mu}$ взвешенного V@R слабо компактно; это вытекает из явного представления этого множества, полученного в [8] (доказательство можно также найти в [36, теорема 4.73] или $[60$, теорема 1.53$])$; это также следует из представления $\mathscr{D}_{\mu}$, полученного в [16, теорема 4.6].

Следующий пример показывает, что условие $X \in L_{\mathrm{s}}^{1}(\mathscr{D})$ в предложении 2.2 нельзя заменить условием $X \in L_{\mathrm{w}}^{1}(\mathscr{D})$.

П р и м е р 2.3. Пусть $\Omega=[0,1]$, мера $\mathbf{P}$ - мера Лебега. Рассмотрим $Z_{n}=\sqrt{n} I_{[0,1 / n]}+1-1 / \sqrt{n}, n \in \mathbf{N}$. Тогда $Y_{n}:=Z_{n}-1 \stackrel{L^{1}}{\longrightarrow} 0$, и поэтому множество

$$
\mathscr{D}=\left\{1+\sum_{n=1}^{\infty} a_{n} Y_{n}: a_{n} \geqslant 0, \sum_{n=1}^{\infty} a_{n} \leqslant 1\right\}
$$

выпукло, $L^{1}$-замкнуто и равномерно интегрируемо. Значит, $\mathscr{D}$ слабо компактно. Теперь рассмотрим $X(\omega)=-1 / \sqrt{\omega}$. Тогда $\mathbf{E}_{\mathbf{p}} Z_{n} X=-4+$ $2 / \sqrt{n}$. Таким образом, $\inf _{\mathbf{Q} \in \mathscr{D}} \mathbf{E}_{\mathbf{Q}} X=-4$. В то же время не существует меры $\mathrm{Q} \in \mathscr{D}$ такой, что $\mathbf{E}_{\mathbf{Q}} X=-4$.

2.4. Размещение капитала. Пусть $(\Omega, \mathscr{F}, \mathbf{P})$ - вероятностное пространство, $u$ - когерентная функция полезности с определяющим множеством $\mathscr{D}$, и пусть $X^{1}, \ldots, X^{d} \in L_{\mathrm{w}}^{1}(\mathscr{D})$ - дисконтированные прибыли, получаемые разными отделами некоторой фирмы за единичный период времени (под прибылью здесь понимается разность конечного капитала и начального капитала, так что $X^{i}$ принимают как положительные, так и отрицательные значения). Будем использовать обозначение $X=\left(X^{1}, \ldots, X^{d}\right)$.

Неформально, задача размешения капитала состоит в следуюшем: как общий риск $\rho\left(\sum_{i} X^{i}\right)$ распределяется между отделами $1, \ldots, d$ ? Иными словами, мы ищем вектор $\left(x^{1}, \ldots, x^{d}\right)$ такой, что $x^{i}$ означает часть риска, которую вносит $i$-й отдел. Набор $x^{i}=\rho\left(X^{i}\right)$ не подходит, так как $\sum_{i} \rho\left(X^{i}\right) \neq \rho\left(\sum_{i} X^{i}\right)$. Следующее определение размещения капитала взято из [22, раздел 9]. Оно тесно связано с коалиционными играми (cM. [26]). 
Задача размещения капитала: найти $x^{1}, \ldots, x^{d} \in \mathbf{R}$ такие, что

$$
\begin{gathered}
\sum_{i=1}^{d} x^{i}=u\left(\sum_{i=1}^{d} X^{i}\right) \\
\forall h^{1}, \ldots, h^{d} \in \mathbf{R}_{+} \quad \sum_{i=1}^{d} h^{i} x^{i} \geqslant u\left(\sum_{i=1}^{d} h^{i} X^{i}\right) .
\end{gathered}
$$

Будем называть решение этой задачи размещением полезности между $X^{1}, \ldots, X^{d}$. Размещение капитала определяется как размещение полезности с обратным знаком.

С финансовой точки зрения, $-x^{i}-$ вклад $i$-го отдела в общий риск фирмы, или, что эквивалентно, капитал, который нужно выделить этому отделу. Для того чтобы проиллюстрировать смысл условия (2.8), рассмотрим пример: $h^{i}=I(i \in J)$, где $J$ - подмножество $\{1, \ldots, d\}$. Тогда (2.8) преврашается в условие $\sum_{i \in J}\left(-x^{i}\right) \leqslant \rho\left(\sum_{i \in J} X^{i}\right)$, которое означает, что капитал, выделенный любой части фирмы, не превышает общего риска, присущего этой части (неравенство (2.8) утверждает то же для «взвешенных» частей фирмы).

Введем обозначение $G=\operatorname{cl}\left\{\mathbf{E}_{\mathrm{Q}} X: \mathrm{Q} \in \mathscr{D}\right\}$, где «cl» означает замыкание. Заметим, что $G$ выпукло и компактно. Будем называть его генерируюшим множеством или просто генератором для $X$ и $u$. Роль этого понятия видна из равенства

$$
\begin{aligned}
u(\langle h, X\rangle) & =\inf _{\mathbf{Q} \in \mathscr{D}} \mathbf{E}_{\mathbf{Q}}\langle h, X\rangle=\inf _{\mathbf{Q} \in \mathscr{D}}\left\langle h, \mathbf{E}_{\mathbf{Q}} X\right\rangle \\
& =\min _{x \in G}\langle h, x\rangle, \quad h \in \mathbf{R}^{d},
\end{aligned}
$$

где $\langle x, y\rangle=\sum_{i=1}^{d} x^{i} y^{i}$ обозначает скалярное произведение. Заметим, что выражение справа - фундаментальный объект выпуклого анализа, а именно опорная функция множества $G$.

Теорема 2.2. Множество $U$ размещений полезности между $X^{1}, \ldots, X^{d}$ имеет вид

$$
U=\underset{x \in G}{\arg \min }\langle e, x\rangle
$$

где $е=(1, \ldots, 1)$. Кроме того, для любого размещения полезности $x$ справедливо неравенство

$$
\forall h^{1}, \ldots, h^{d} \in \mathbf{R} \quad \sum_{i=1}^{d} h^{i} x^{i} \geqslant u\left(\sum_{i=1}^{d} h^{i} X^{i}\right) .
$$

Если к тому же $X^{1}, \ldots, X^{d} \in L_{\mathbf{s}}^{1}(\mathscr{D})$, а $\mathscr{D}$ слабо замкнуто, то

$$
U=\left\{\mathbf{E}_{\mathrm{Q}} X: \mathbf{Q} \in \mathscr{X}_{\mathscr{D}}\left(\sum_{i=1}^{d} X^{i}\right)\right\} \text {. }
$$


Д ок а з а т ль с т в о. (Доказательство иллюстрируется на рис. 1.) Для $h \in \mathbf{R}^{d}$ положим

$$
\begin{aligned}
L(h) & =\left\{x \in \mathbf{R}^{d}:\langle h, x\rangle=\min _{y \in G}\langle h, y\rangle\right\}, \\
M(h) & =\left\{x \in \mathbf{R}^{d}:\langle h, x\rangle \geqslant \min _{y \in G}\langle h, y\rangle\right\} .
\end{aligned}
$$

Из (2.9) видно, что множество точек $x \in \mathbf{R}^{d}$, удовлетворяющих условию (2.7), есть $L(e)$. Множество точек $x$, удовлетворяющих условию $(2.8)$, есть $\bigcap_{h \in \mathbf{R}_{+}^{d}} M(h)=G+\mathbf{R}_{+}^{d}$. Множество точек $x$, удовлетворяющих условию (2.11), есть $\bigcap_{h \in \mathbf{R}^{d}} M(h)=G$. Это доказывает $(2.10)$ и (2.11). Далее, множество $\left\{\mathbf{E}_{\mathbf{Q}} X: \mathbf{Q} \in \mathscr{D}\right\}$ замкнуто (это доказывается так же, как предложение 2.2). Теперь равенство (2.12) вытекает из (2.10) и определения $\mathscr{X}_{\mathscr{D}}$. Теорема 2.2 доказана.

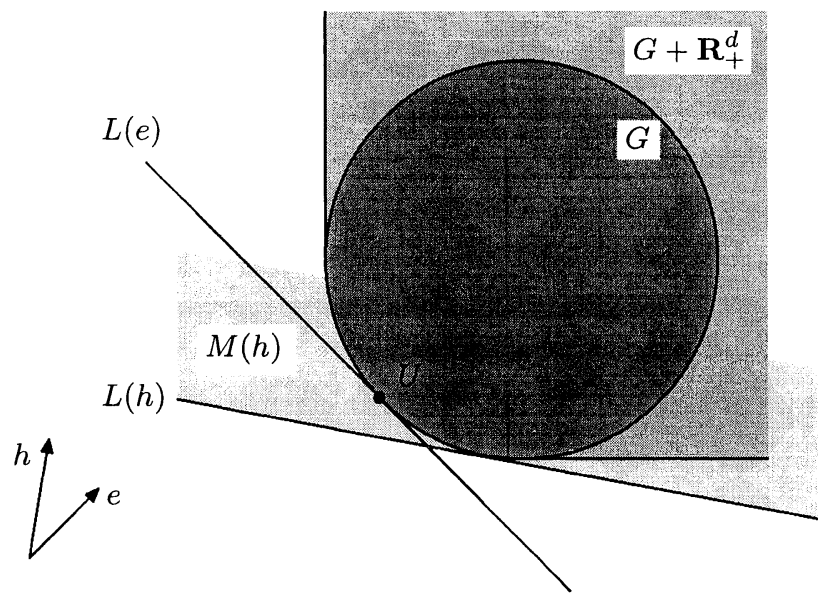

Рис. 1. Решение задачи размешения капитала

Если $G$ строго выпукло (т.е. его внутренность непуста, а граница не содержит никакого интервала), то размещение капитала единственно. Однако в общем случае единственность может нарушаться, как показывает пример ниже.

П р и м е р 2.4. Пусть $d=2$ и $X^{2}=-X^{1}$. Тогда $G$ - интервал с концевыми точками $\left(u\left(X^{1}\right),-u\left(X^{1}\right)\right)$ и $\left(-u\left(-X^{1}\right), u\left(-X^{1}\right)\right)$. В этом примере $U=G$.

Найдем теперь решение задачи размещения капитала в гауссовском случае.

П р и м е р 2.5. Пусть $X$ имеет гауссовское распределение со средним $a$ и ковариационной матрицей $C$. Пусть $u-$ инвариантная по распределению когерентная функция полезности, т.е. $u(X)$ зависит только 
от распределения $X$ (заметим, что хвостовой $\mathrm{V} @ \mathrm{R}$ и взвешенный $\mathrm{V} @ \mathrm{R}$ удовлетворяют этому свойству); будем также предполагать, что $u$ конечна на гауссовских случайных величинах.

Тогда существует константа $\gamma>0$ такая, что для любой гауссовской случайной величины $\xi$ со средним $m$ и дисперсией $\sigma^{2}$ имеем $u(\xi)=m-\gamma \sigma$. Обозначим через $L$ образ $\mathbf{R}^{d}$ при отображении $x \mapsto C x$. Тогда корректно определено обратное отображение $C^{-1}: L \rightarrow L$. Легко видеть, что

$$
G=a+\left\{C^{1 / 2} x:\|x\| \leqslant \gamma\right\}=a+\left\{y \in L:\left\langle y, C^{-1} y\right\rangle \leqslant \gamma^{2}\right\} .
$$

Положим $e=(1, \ldots, 1)$ и предположим, что $C e \neq 0$. В этом случае размещение полезности $x_{0}$ между $X^{1}, \ldots, X^{d}$ определено однозначно. Для того чтобы его найти, заметим, что для любого $y \in L$ такого, что

$$
\left.\frac{d}{d \varepsilon}\right|_{\varepsilon=0}\left\langle x_{0}-a+\varepsilon y, C^{-1}\left(x_{0}-a+\varepsilon y\right)\right\rangle=0
$$

выполнено равенство $\langle e, y\rangle=0$. Это означает, что $C^{-1}\left(x_{0}-a\right)=\alpha \mathrm{pr}_{L} e$ с некоторой константой $\alpha\left(\mathrm{pr}_{L}\right.$ обозначает ортогональную проекцию на $L$ ). Итак, $x_{0}=a+\alpha C e$. Поскольку точка $x_{0}$ должна принадлежать относительной внутренности множества $G$ (т.е. внутренности в относительной топологии $a+L)$, получаем $\left\langle x_{0}-a, C^{-1}\left(x_{0}-a\right)\right\rangle=\gamma^{2}$, т.е. $\alpha=-\gamma\langle e, C e\rangle^{-1 / 2}$. В результате размещение полезности между $X^{1}, \ldots, X^{d}$ имеет вид $a-\gamma\langle e, C e\rangle^{-1 / 2} C e$.

Предположим теперь, что $C e=0$. Это значит, что $e$ ортогонален $L$, и тогда множеством размещений полезности между $X^{1}, \ldots, X^{d}$ служит $G$.

Отметим, что в этом примере множество размещений полезности зависит от $u$ весьма слабо, а именно, зависит лишь от $\gamma$.

2.5. Риск-вклад. Пусть $(\Omega, \mathscr{F}, \mathbf{P})$ - вероятностное пространство, $u$ - когерентная функция полезности с определяющим множеством $\mathscr{D}$, а $Y \in L^{0}$ - дисконтированная прибыль, получаемая некоторой фирмой.

Такая фирма оценивает риск любого портфеля, приносящего некоторую прибыль $X$, не как $\rho(X)$, а как $\rho(Y+X)-\rho(Y)$. Ниже мы даем определение риск-вклада $\rho^{\mathrm{c}}(X ; Y)$ таким образом, что $\rho(\cdot ; Y)$ - когерентная мера риска и $\rho^{\mathrm{c}}(X ; Y) \approx \rho(Y+X)-\rho(Y)$, если случайная величина $X$ мала по сравнению с $Y$ (точное утверждение содержится в теореме 2.3).

О п р е д е л е н и е 2.5. Вклад полезности $X$ в $Y$ определим как

$$
u^{\mathrm{c}}(X ; Y)=\inf _{\mathbf{Q} \in \mathscr{X}_{\mathscr{D}}(Y)} \mathbf{E}_{\mathbf{Q}} X .
$$

Риск-вклад $X$ в $Y$ определим как $\rho^{\mathrm{c}}(X ; Y)=-u^{\mathrm{c}}(X ; Y)$. 
Вклад полезности является когерентной функцией полезности при условии, что $\mathscr{X}_{\mathscr{D}}(Y) \neq \varnothing$. Например, это так, если $\mathscr{D}$ слабо компактно и $Y \in L_{\mathrm{s}}^{1}(\mathscr{D})$.

Если $\mathscr{D}$ слабо компактно и $X, Y \in L_{\mathrm{s}}^{1}(\mathscr{D})$, то по теореме 2.2

$$
\begin{gathered}
u^{\mathrm{c}}(X ; Y)=\inf \left\{x^{1}:\left(x^{1}, x^{2}\right)\right. \text { является размещением полезности } \\
\text { между } X, Y-X\} .
\end{gathered}
$$

Используя эту формулу, мы можем определить риск-вклад при следующих ограничениях: $\mathscr{D}$ произвольно, а $X, Y \in L_{\mathrm{w}}^{1}(\mathscr{D})$.

Если $\mathscr{D}$ слабо компактно, $X^{1}, \ldots, X^{d} \in L_{\mathrm{s}}^{1}(\mathscr{D})$, а множество $\mathscr{X}_{\mathscr{D}}\left(\sum_{i} X^{i}\right)$ одноточечно, то (ввиду теоремы 2.2 ) размещение полезности между $X^{1}, \ldots, X^{d}$ единственно и имеет вид

$$
\left(u^{\mathrm{c}}\left(X^{1} ; \sum_{i=1}^{d} X^{i}\right), \ldots, u^{\mathrm{c}}\left(X^{d} ; \sum_{i=1}^{d} X^{i}\right)\right) .
$$

Это указывает на правильность введенного определения. Другим утверждением, поддерживающим это определение, служит следующая теорема.

Теорема 2.3. $\quad$ Если $\mathscr{D}$ слабо компактно и $X, Y \in L_{\mathrm{s}}^{1}(\mathscr{D})$, mo

$$
u^{\mathrm{c}}(X ; Y)=\lim _{\varepsilon \downarrow 0} \varepsilon^{-1}(u(Y+\varepsilon X)-u(Y))
$$

Д о к а з а т е л с т в о. (Доказательство иллюстрируется на рис. 2.)

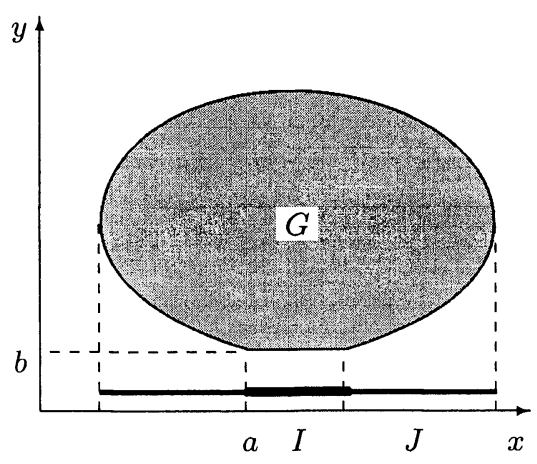

Pис. 2

Рассмотрим генератор $G=\operatorname{cl}\left\{\mathbf{E}_{\mathbf{Q}}(X, Y): \mathbf{Q} \in \mathscr{D}\right\}$ и положим $b=$ $\inf \{y$ : сушествует $x$ такое, что $(x, y) \in G\}, I=\{x:(x, b) \in G\}, J=\{x$ : существует $y$ такое, что $(x, y) \in G\}, a=\inf \{x: x \in I\}$. Заметим, что 
$u^{\mathrm{c}}(X ; Y)=a$. Минимум $\min _{(x, y) \in G}\langle(\varepsilon, 1),(x, y)\rangle$ достигается в некоторой точке $(a(\varepsilon), b(\varepsilon))$. Имеем $a(\varepsilon) \leqslant a, b(\varepsilon) \geqslant b$ и $(a(\varepsilon), b(\varepsilon)) \rightarrow(a, b)$ при $\varepsilon \downarrow 0$. Далее, $\varepsilon a(\varepsilon)+b(\varepsilon) \leqslant \varepsilon a+b$, откуда $0 \leqslant b(\varepsilon)-b \leqslant \varepsilon(a-a(\varepsilon))$. В результате

$$
\begin{aligned}
\lim _{\varepsilon \downarrow 0} \varepsilon^{-1}(u(Y+\varepsilon X)-u(Y)) & =\lim _{\varepsilon \downarrow 0} \varepsilon^{-1}(\varepsilon a(\varepsilon)+b(\varepsilon)-b) \\
& =a+\lim _{\varepsilon \downarrow 0} \varepsilon^{-1}(b(\varepsilon)-b)=a=u^{\mathrm{c}}(X ; Y) .
\end{aligned}
$$

П р и м е р 2.6. (i) Пусть $Y$ - вырожденная случайная величина. В этом случае $\mathscr{X}_{\mathscr{D}}(Y)=\mathscr{D}$, так что $u^{\mathrm{c}}(X ; Y)=u(X)$.

(ii) Пусть $X=\alpha Y$ с $\alpha \in \mathbf{R}_{+}$. Тогда $u^{\mathrm{c}}(X ; Y)=\alpha u(Y)$.

(iii) Пусть $X, Y$ имеют совместное гауссовское распределение со средним $(\mathbf{E} X, \mathbf{E} Y)$ и матрицей ковариаций $C$. Пусть $u$ - инвариантная по распределению когерентная функция полезности, конечная на гауссовских случайных величинах. Тогда существует константа $\gamma>0$ такая, что для любой гауссовской случайной величины $\xi$ со средним $m$ и дисперсией $\sigma^{2}$ выполнено $u(\xi)=m-\gamma \sigma$. Предположим, что $X$ и $Y$ невырождены и $\operatorname{corr}(X, Y) \neq \pm 1$. Из примера 2.5 вытекает, что

$$
\begin{aligned}
u^{\mathrm{c}}(X ; Y) & =\mathbf{E} X-\gamma\left\langle e_{2}, C e_{2}\right\rangle^{-1 / 2} C e_{2}=\mathbf{E} X-\gamma \frac{\operatorname{cov}(X, Y)}{(\mathbf{D} Y)^{1 / 2}} \\
& =\mathbf{E} X+(u(X)-\mathbf{E} X) \operatorname{corr}(X, Y),
\end{aligned}
$$

где $e_{2}=(0,1)$. В частности, если $\mathbf{E} X=\mathbf{E} Y=0$, то

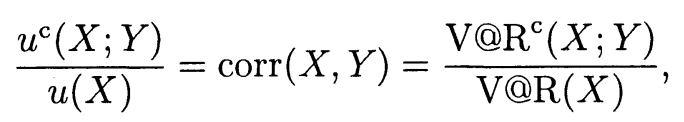

где $\mathrm{V} @ \mathrm{R}^{\mathrm{c}}$ означает V@R-вклад (по поводу определения см. [52, раздел 7]).

\section{3. Нахождение справедливых цен}

3.1. Метод NGD первого рода. Пусть $(\Omega, \mathscr{F}, \mathbf{P})$ - вероятностное пространство, $u$ - когерентная функция полезности со слабо компактным определяющим множеством $\mathscr{D}$, а $A$ - выпуклое подмножество $L^{0}$. С финансовой точки зрения, $A$ - множество различных возможных прибылей, которые можно получить в рассматриваемой модели с использованием различных стратегий (примеры даются в пп. 3.3-3.5). Будем называть $A$ множеством возможных прибылей. Будем предполагать, что $A \mathscr{D}$-согласовано (см. определение 3.2 ниже). В пп. 3.3-3.5 показано, что это предположение автоматически выполнено в естественных моделях.

Прежде всего дадим определение риск-нейтральной меры. Конечно, этот объект является классическим для финансовой математики, но конкретное определение, которое мы используем, взято из [13] (оно адаптировано к $L^{0}$-случаю). 
О п р е д е л е н и е 3.1. Риск-нейтральной мерой называется мера $\mathrm{Q} \in \mathscr{P}$ такая, что $\mathbf{E}_{\mathrm{Q}} X \leqslant 0$ для любого $X \in A$ (используем соглашение $\left.\mathbf{E} X=\mathbf{E} X^{+}-\mathbf{E} X^{-}, \infty-\infty=-\infty\right)$. Множество риск-нейтральных мер будем обозначать $\mathscr{R}$ или $\mathscr{R}(A)$, если возможна неопределенность.

О п р е д е л е н и е 3.2 . Множество $A \mathscr{D}$-согласовано, если существует множество $A^{\prime} \subseteq A \cap L_{\mathrm{s}}^{1}(\mathscr{D})$ такое, что $\mathscr{D} \cap \mathscr{R}=\mathscr{D} \cap \mathscr{R}\left(A^{\prime}\right)$.

О п р е д е л е н и е 3.3. Модель удовлетворяет условию $N G D$ nepвого рода, если не существует $X \in A$ такого, что $u(X)>0$.

Теорема 3.1 (фундаментальная теорема теории расчетов). $M$ дель удовлетворяет условию NGD первого рода тогда и только тогда, когда $\mathscr{D} \cap \mathscr{R} \neq \varnothing$.

Д ок а з а т ль с т в о. Достаточность очевидна. Докажем необходимость. Фиксируем $X_{1}, \ldots, X_{M} \in A^{\prime}$. Из слабой непрерывности отображений $\mathscr{D} \ni \mathbf{Q} \mapsto \mathbf{E}_{\mathbf{Q}} X_{m}$ следует, что множество $G=$ $\left\{\mathbf{E}_{\mathbf{Q}}\left(X_{1}, \ldots, X_{M}\right): \mathbf{Q} \in \mathscr{D}\right\}$ компактно. Очевидно, $G$ выпукло. Допустим, что $G \cap(-\infty, 0]^{M}=\varnothing$. Тогда существуют $h \in \mathbf{R}^{M}$ и $\varepsilon>0$ такие, что $\langle h, x\rangle \geqslant \varepsilon$ для любого $x \in G$ и $\langle h, x\rangle \leqslant 0$ для любого $x \in(-\infty, 0]^{M}$. Следовательно, $h \in \mathbf{R}_{+}^{M}$. Без ограничения общности можно считать, что $\sum_{m} h_{m}=1$. Тогда $X=\sum_{m} h_{m} X_{m} \in A$ и $\mathbf{E}_{\mathbf{Q}} X \geqslant \varepsilon$ для любого $\mathbf{Q} \in \mathscr{D}$, так что $u(X)>0$.

Полученное противоречие показывает, что для любых $X_{1}, \ldots, X_{M} \in$ $A^{\prime}$ множество

$$
B\left(X_{1}, \ldots, X_{M}\right)=\left\{\mathbf{Q} \in \mathscr{D}: \mathbf{E}_{\mathbf{Q}} X_{m} \leqslant 0 \text { для любого } m=1, \ldots, M\right\}
$$

непусто. Поскольку $X_{m} \in L_{\mathrm{s}}^{1}(\mathscr{D})$, то отображение $\mathscr{D} \ni \mathbf{Q} \mapsto \mathbf{E}_{\mathbf{Q}} X_{m}$ слабо непрерывно, и поэтому $B\left(X_{1}, \ldots, X_{M}\right)$ слабо замкнуто. Далее, любое непустое пересечение множеств такого вида непусто. В силу слабой компактности $\mathscr{D}$ существует мера $\mathrm{Q}$ принадлежащая всем таким множествам $B$. Тогда $\mathbf{E}_{\mathbf{Q}} X \leqslant 0$ для любого $X \in A^{\prime}$, что означает, что $\mathrm{Q} \in \mathscr{D} \cap \mathscr{R}\left(A^{\prime}\right)$. Поскольку $A \mathscr{D}$-согласовано, то $\mathrm{Q} \in \mathscr{D} \cap \mathscr{R}$. Теорема 3.1 доказана.

3 а м е ч а н и я. (i) В отличие от фундаментальных теорем расчетов, основанных на условии безарбитражности и его усилениях (см. [13], [23], [24]), нам не нужно брать замыкание $A$ при определении NGD. По сути, в основе нашей фундаментальной теоремы лежит компактность множества $\mathscr{D}$.

(ii) При $\mathscr{D}=\mathscr{P}$ условие NGD означает, что не существует $X \in A$ c ess inf ${ }_{\omega} X(\omega)>0$. Это почти совпадает с условием безарбитражности. Однако в этом случае $\mathscr{D}$ не является равномерно интегрируемым и теорема 3.1 нарушается. Действительно, пусть $A=\{h X: h \in \mathbf{R}\}$, где $X$ имеет равномерное распределение на $[0,1]$. Тогда NGD выполнено, в то время как $\mathscr{R}=\varnothing$. 
Пусть теперь $F \in L^{0}$ - дисконтированная функция выплат некоторого платежного поручения.

О п р е д е лен и е 3.4. Справедливой $N G D$-ценой поручения $F$ назовем вещественное число $x$ такое, что расширенная модель $(\Omega, \mathscr{F}, \mathbf{P}, \mathscr{D}, A+\{h(F-x): h \in \mathbf{R}\})$ удовлетворяет условию NGD. Mножество справедливых цен обозначим $I_{\mathrm{NGD}}(F)$.

Следствие 3.1 (интервал справедливых цен). Для $F \in L_{\mathrm{s}}^{1}(\mathscr{D})$

$$
I_{\mathrm{NGD}}(F)=\left\{\mathbf{E}_{\mathbf{Q}} F: \mathbf{Q} \in \mathscr{D} \cap \mathscr{R}\right\} .
$$

Д о к а з а т е л ь с т в о. Обозначим $\{h(F-x): h \in \mathbf{R}\}$ через $A(x)$. Очевидно, $A+A(x) \mathscr{D}$-согласовано (для доказательства этого достаточно рассмотреть $\left.A^{\prime}+A(x)\right)$. Из теоремы 3.1 следует, что $x \in I_{\mathrm{NGD}}(F)$ тогда и только тогда, когда $\mathscr{D} \cap \mathscr{R}(A+A(x)) \neq \varnothing$. Легко видеть, что $\mathbf{Q} \in$ $\mathscr{R}(A+A(x))$ тогда и только тогда, когда $\mathbf{Q} \in \mathscr{R}$ и $\mathbf{E}_{\mathbf{Q}} F=x$. Это завершает доказательство.

3 а м е ч а н и е. В отличие от безарбитражных интервалов цен, интервалы справедливых NGD-цен замкнуты (это следует из слабой непрерывности отображения $\left.\mathscr{D} \cap \mathscr{R} \mapsto \mathrm{E}_{\mathbf{Q}} F\right)$.

В завершение этого пункта рассмотрим возможные типы задания множества $\mathscr{D}$. Во-первых, $\mathscr{D}$ может быть определяющим множеством когерентной функции полезности, такой как хвостовой V@R или взвешенный V@R. Taкже $\mathscr{D}$ может отвечать выпуклой комбинации или минимуму нескольких когерентных функций полезности. Но кроме того $\mathscr{D}$ может происходить из классической максимизации полезности, как показывает пример ниже.

П р и м е р 3.1. Пусть $\mathbf{P}_{1}, \ldots, \mathbf{P}_{N}$ - набор вероятностных мер, $U_{1}, \ldots, U_{N}$ - набор классических функций полезности (т.е. гладких вогнутых возрастающих функций), а $W_{1}, \ldots, W_{N}$ - набор случайных величин. С финансовой точки зрения, $\mathbf{P}_{n}, U_{n}$ и $W_{n}$ - субъективная вероятность, функция полезности и конечный капитал $n$-го участника рынка соответственно. Рассмотрим меру $\mathbf{Q}_{n}=c_{n} U_{n}^{\prime}\left(W_{n}\right) \mathbf{P}_{n}$, где $c_{n}$ - нормирующий множитель. Тогда для любой возможной прибыли $X \in L^{0}$ имеем

$$
\left.\frac{d}{d \varepsilon}\right|_{\varepsilon=0} U_{n}\left(W_{n}+\varepsilon X\right)=\mathbf{E}_{\mathbf{P}_{n}} U_{n}^{\prime}\left(W_{n}\right) X=\mathbf{E}_{\mathbf{Q}_{n}} c_{n}^{-1} X
$$

(предполагается, что все ожидания существуют и что интегрирование можно переставлять с дифференцированием). Итак, операция, приносящая прибыль $\varepsilon X$ с малым $\varepsilon>0$, привлекательна для $n$-го участника тогда и только тогда, когда $\mathbf{E}_{\mathbf{Q}_{n}} X>0$, так что $\mathbf{Q}_{n}$ можно назвать оценочной мерой $n$-го участника. Возьмем $\mathscr{D}=\operatorname{conv}\left(\mathbf{Q}_{1}, \ldots, \mathbf{Q}_{N}\right)$ и рассмотрим соответствуюшую когерентную функцию полезности $u$. Тогда $u(X)>0$, тогда и только тогда, когда $\mathbf{E}_{\mathrm{Q}_{n}} X>0$ для любого $n$. 
Ввиду (3.1) это означает, что прибыль $\varepsilon X$ с некоторым $\varepsilon>0$ привлекательна для любого участника рынка (это сходно с понятием строго приемлемой возможности, введенным в [9]). Итак, в данном примере условие NGD означает отсутствие операций, привлекательных для любого участника.

3.2. Метод NGD второго рода. Пусть $(\Omega, \mathscr{F}, \mathbf{P})$ - вероятностное пространство, $\mathscr{R} \mathscr{D} \subset \mathscr{P}$ - выпуклое слабо компактное множество, $\mathscr{P} \mathscr{D}-L^{1}$-замкнутое выпуклое подмножество $\mathscr{R} \mathscr{D}$, а $A-$ $\mathscr{R} \mathscr{D}$-согласованное выпуклое подмножество $L^{0}$. Будем называть $\mathscr{P} \mathscr{D}$ множеством, определяющим прибыльность. Это означает, что прибыльность операции, приносящей случайный доход $X$, определяется как $\inf _{\mathbf{Q} \in \mathscr{P} \mathscr{D}} \mathbf{E}_{\mathbf{Q}} X$. Будем называть $\mathscr{R} \mathscr{D}$ множеством, определяюшим риск. Это означает, что риск операции, приносящей случайный доход $X$, определяется как $-\inf _{\mathbf{Q} \in \mathscr{D}} \mathbf{E}_{\mathbf{Q}} X$. Канонический пример: $\mathscr{P} \mathscr{D}=\{\mathbf{P}\}$, a $\mathscr{R} \mathscr{D}$ - определяющее множество когерентной функции полезности. Тот факт, что $\mathscr{P} \mathscr{D}$ не обязано быть одноточечным, отвечает неоднозначности исходной вероятностной меры, или, другими словами, модельному риску. Финансовая интерпретация множества $A$ та же, что и выше. Также зафиксируем положительное число $R$, означающее верхний порог возможных скорректированных на риск доходностей.

О п р е д е л е н и е 3.5. Скорректированная на риск доходность (будем использовать англоязычную аббревиатуру RAROC - Risk Adjusted Return on Capital) для $X \in L^{0}$ определяется следующим образом:

$$
\operatorname{RAROC}(X)= \begin{cases}+\infty, & \text { если } \inf _{\mathbf{Q} \in \mathscr{P} \mathscr{D}} \mathbf{E}_{\mathbf{Q}} X>0 \text { и } \\ \frac{\inf _{\mathbf{Q} \in \mathscr{P} \mathscr{D}} \mathbf{E}_{\mathbf{Q}} X}{-\inf _{\mathbf{Q} \in \mathscr{R} \mathscr{D}} \mathbf{E}_{\mathbf{Q}} X} & \text { иначе }\end{cases}
$$

с соглашением $\frac{0}{0}=0, \frac{\infty}{\infty}=0$.

О п р ед е л ен и е 3.6. Модель удовлетворяет условию $N G D$ второго рода, если не существует $X \in A$ такого, что $\operatorname{RAROC}(X)>R$.

Слгдствие 3.2 (фундаментальная теорема теории расчетов). Moдель удовлетворяет условию NGD второго рода тогда и только тогда, когда

$$
\left(\frac{1}{1+R} \mathscr{P} \mathscr{D}+\frac{R}{1+R} \mathscr{R} \mathscr{D}\right) \cap \mathscr{R} \neq \varnothing .
$$

Д оказ а т е ль с т в о. Рассмотрим сначала случай $R>0$. Тогда для любого $X \in L^{0}$

$\operatorname{RAROC}(X)>R \Longleftrightarrow \inf _{\mathbf{Q} \in \mathscr{P} \mathscr{D}} \mathbf{E}_{\mathbf{Q}} X+R \inf _{\mathbf{Q} \in \mathscr{R} \mathscr{D}} \mathbf{E}_{\mathbf{Q}} X>0 \Longleftrightarrow \inf _{\mathbf{Q} \in \mathscr{D}} \mathbf{E}_{\mathbf{Q}} X>0$ 
где $\mathscr{D}=(1+R)^{-1} \mathscr{P} \mathscr{D}+R(1+R)^{-1} \mathscr{R} \mathscr{D}$. Очевидно, $\mathscr{D}$ слабо компактно, а $A \mathscr{D}$-согласовано (заметим, что $L_{\mathrm{s}}^{1}(\mathscr{D})=L_{\mathrm{s}}^{1}(\mathscr{R} \mathscr{D})$ ). Теперь утверждение вытекает из теоремы 3.1 .

Рассмотрим случай $R=0$. В этом случае достаточность очевидна, и нужно проверить лишь необходимость. Выберем множество $A^{\prime} \subseteq A \cap L_{\mathrm{s}}^{1}(\mathscr{R} \mathscr{D})$ такое, что $\mathscr{R} \mathscr{D} \cap \mathscr{R}=\mathscr{R} \mathscr{D} \cap \mathscr{R}\left(A^{\prime}\right)$. Для любого $X \in \operatorname{conv} A^{\prime}$ имеем $\inf _{\mathbf{Q} \in \mathscr{P} \mathscr{D}} \mathbf{E}_{\mathbf{Q}} X \leqslant 0$. Повторяя рассуждения из доказательства теоремы 3.1 , получаем $\mathscr{P} \mathscr{D} \cap \mathscr{R} \neq \varnothing$.

О п р е д е л е н и е 3.7. Справедливой $N G D$-ченой платежного поручения $F$ назовем вещественное число $x$ такое, что модель $(\Omega, \mathscr{F}, \mathbf{P}, \mathscr{P} \mathscr{D}, \mathscr{R} \mathscr{D}, A+\{h(F-x): h \in \mathbf{R}\})$ удовлетворяет условию NGD. Множество справедливых NGD-цен будем обозначать через $I_{\mathrm{NGD}}(F)$.

Следствие 3.3 (интервал справедливых цен). Для $F \in L_{\mathrm{s}}^{1}(\mathscr{D})$

$$
I_{\mathrm{NGD}}(F)=\left\{\mathbf{E}_{\mathbf{Q}} F: \mathbf{Q} \in\left(\frac{1}{1+R} \mathscr{P} \mathscr{D}+\frac{R}{1+R} \mathscr{R} \mathscr{D}\right) \cap \mathscr{R}\right\} .
$$

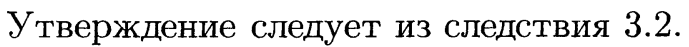

Сравним теперь возможные практические применения двух предложенных форм метода NGD.

$\Pi$ р и м е р 3.2. Пусть $\mathscr{P} \mathscr{D}=\{\mathbf{P}\}, \mathscr{D}$ - определяющее множество хвостового V@R порядка 0.05 (это типичный выбор для меры риска), $A=0$ (т.е. рынок отсутствует), $R=0.1$ (это типичное значение RAROC для периода в 1 год), а $F$ - гауссовская случайная величина со средним 0 и дисперсией 1. Тогда агент, использующий метод NGD первого рода, будет просить за такое платежное поручение сумму

$$
\sup _{\mathbf{Q} \in \mathscr{D}} \mathbf{E}_{\mathbf{Q}} F=-u_{0.05}(-F)=-\int_{-\infty}^{\Phi^{-1}(0.05)} \frac{1}{\sqrt{2 \pi}} x e^{-x^{2} / 2} d x=2.06
$$

( $\Phi$ - функция распределения стандартного нормального закона), что является неприемлемо высокой ценой для покупателя такого контракта (действительно, он будет в минусе в $98 \%$ случаев). В то же время агент, использующий метод NGD второго рода, будет просить за такое поручение сумму

$$
\sup _{\mathbf{Q} \in(1+R)^{-1} \mathbf{P}+R(1+R)^{-1} \mathscr{D}} \mathbf{E}_{\mathbf{Q}} F=\frac{R}{1+R} \sup _{\mathbf{Q} \in \mathscr{D}} \mathbf{E}_{\mathbf{Q}} F=\frac{0.1 \cdot 2.06}{1.1}=0.19,
$$

что является разумной ценой.

Итак, если в качестве меры риска берется одна из тех мер, которые реально используются для оценки риска, то нужно использовать метод NGD второго рода. Для метода же NGD первого рода нужно сразу брать гораздо более «умеренные» меры риска (например, хвостовой V@R c параметром, близким к 1). 
3.3. Статическая модель с конечным числом активов. Рассмотрим модель предыдущего пункта с $A=\left\{\left\langle h, S_{1}-S_{0}\right\rangle: h \in \mathbf{R}^{d}\right\}$, где $S_{0} \in \mathbf{R}^{d}$, а $S_{1}^{1}, \ldots, S_{1}^{d} \in L_{\mathrm{s}}^{1}(\mathscr{R} \mathscr{D})$. С финансовой точки зрения, $S_{n}^{i}-$ дисконтированная цена $i$-го актива в момент $n$.

Очевидно, в этой модели $A \mathscr{R} \mathscr{D}$-согласовано и $\mathscr{R} \mathscr{D} \cap \mathscr{R}=\mathscr{R} \mathscr{D} \cap \mathscr{M}$, где $\mathscr{M}$ - множество мартингальных мер:

$$
\mathscr{M}=\left\{\mathbf{Q} \in \mathscr{P}: \mathbf{E}_{\mathbf{Q}}\left|S_{1}\right|<\infty \quad \text { и } \quad \mathbf{E}_{\mathbf{Q}} S_{1}=S_{0}\right\}
$$

3 а м е ч а н и е. Имеем $\mathscr{M} \subseteq \mathscr{R}$, но обратное включение может нарушаться. Действительно, пусть $d=1$, а $S_{1}$ удовлетворяет условию $\mathbf{E}_{\mathbf{P}} S_{1}^{+}=\mathbf{E}_{\mathbf{P}} S_{1}^{-}=\infty$. Тогда $\mathbf{P} \in \mathscr{R}$, в то время как $\mathbf{P} \notin \mathscr{M}$.

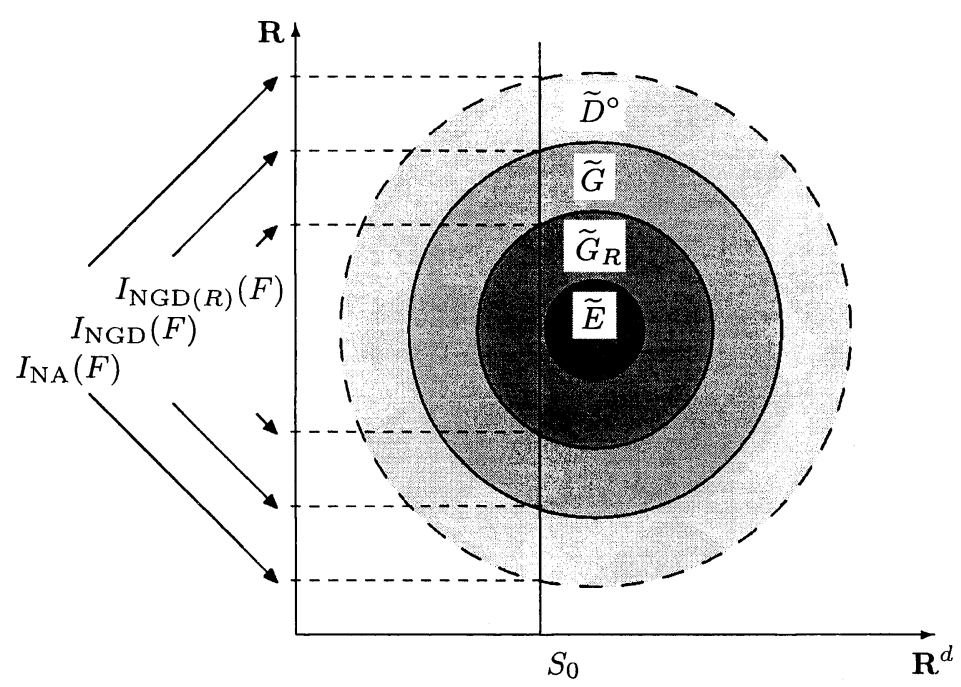

Рис. 3. Геометрическое представление интервалов справедливых цен для разных методов

Дадим теперь геометрическую интерпретацию теоремы 3.1 и следствия 3.2. Для этого нам достаточно лишь предполагать, что $\mathscr{P} \mathscr{D} \subseteq$ $\mathscr{R} \mathscr{D} \subseteq \mathscr{P}$ - выпуклые множества и $S_{1}^{i} \in L_{\mathrm{w}}^{1}(\mathscr{R} \mathscr{D})$ для любого $i$. Введем обозначения (см. рис. 3 )

$$
\begin{aligned}
E & =\operatorname{cl}\left\{\mathbf{E}_{\mathbf{Q}} S_{1}: \mathbf{Q} \in \mathscr{P} \mathscr{D}\right\}, & & G=\operatorname{cl}\left\{\mathbf{E}_{\mathbf{Q}} S_{1}: \mathbf{Q} \in \mathscr{R} \mathscr{D}\right\}, \\
G_{R} & =\frac{1}{1+R} E+\frac{R}{1+R} G, & & D=\text { convsupp Law } S_{1},
\end{aligned}
$$

где «supp» обозначает носитель, и обозначим $D^{\circ}$ относительную внутренность $D$ (т.е. внутренность в относительной топологии наименьшего 
аффинного пространства, содержащего $D)$. Из равенств

$$
\begin{aligned}
\inf _{\mathbf{Q} \in \mathscr{P} \mathscr{D}} \mathbf{E}_{\mathbf{Q}}\left\langle h, S_{1}-S_{0}\right\rangle & =\inf _{x \in E}\left\langle h, x-S_{0}\right\rangle, \\
\inf _{\mathbf{Q} \in \mathscr{R} \mathscr{D}} \mathbf{E}_{\mathbf{Q}}\left\langle h, S_{1}-S_{0}\right\rangle & =\inf _{x \in G}\left\langle h, x-S_{0}\right\rangle
\end{aligned}
$$

вытекают импликации

$$
\begin{aligned}
& \text { условие NGD второго рода } \Longleftrightarrow S_{0} \in G_{R}, \\
& \text { условие NGD первого рода } \Longleftrightarrow S_{0} \in G, \\
& \text { условие NA } \Longleftrightarrow S_{0} \in D^{\circ},
\end{aligned}
$$

где для метода NGD первого рода берется множество $\mathscr{D}=\mathscr{R} \mathscr{D}$ (последняя эквивалентность - известный результат теории арбитража; см. [64, гл. $\mathrm{V}, \S 2 \mathrm{e}])$.

Пусть теперь $F \in L_{\mathrm{w}}^{1}(\mathscr{R} \mathscr{D})$ - дисконтированная функция выплат некоторого платежного поручения. Пусть $\widetilde{E}, \widetilde{G}, \widetilde{G}_{R}, \widetilde{D}$ и $\widetilde{D}^{\circ}$ - аналоги множеств $E, G, G_{R}, D$ и $D^{\circ}$, определенные для $\widetilde{S}_{1}=\left(S_{1}^{1}, \ldots, S_{1}^{d}, F\right)$ вместо $S_{1}$. Пусть $I_{\mathrm{NGD}}(F)$ - интервал справедливых цен для метода NGD первого рода, $I_{\mathrm{NGD}(R)}(F)$ - интервал справедливых цен для метода NGD второго рода, а $I_{\mathrm{NA}}(F)$ - интервал безарбитражных цен. Тогда

$$
\begin{aligned}
I_{\mathrm{NGD}(R)}(F) & =\left\{x:\left(S_{0}, x\right) \in \widetilde{G}_{R}\right\}, \quad I_{\mathrm{NGD}}(F)=\left\{x:\left(S_{0}, x\right) \in \widetilde{G}\right\}, \\
I_{\mathrm{NA}}(F) & =\left\{x:\left(S_{0}, x\right) \in \widetilde{D}^{\circ}\right\} .
\end{aligned}
$$

П р и м е р 3.3. Пусть $S_{1}$ имеет гауссовское распределение со средним $a$ и матрицей ковариаций $C$. Пусть $\mathscr{P} \mathscr{D}=\{\mathbf{P}\}$, а $\mathscr{R} \mathscr{D}$ - определяющее множество для инвариантной по распределению когерентной функции полезности $u$, принимающей конечные значения на гауссовских случайных величинах. Пусть $F$ такова, что вектор $\left(S_{1}^{1}, \ldots, S_{1}^{d}, F\right)$ гауссовский. Положим $c=\operatorname{cov}\left(S_{1}, F\right)$ (пользуемся векторной формой обозначений).

Существует вектор $b \in \mathbf{R}^{d}$ такой, что $C b=c$. Можем представить $F$ в виде $F=\left\langle b, S_{1}-a\right\rangle+\mathbf{E} F+\widetilde{F}$. Тогда $\mathbf{E} \widetilde{F}=0$ и $\operatorname{cov}\left(\widetilde{F}, S_{1}\right)=0$, так что $\widetilde{F}$ и $S_{1}$ независимы. Заметим, что

$$
\sigma^{2}:=\mathbf{D} \widetilde{F}=\mathbf{D} F-\mathbf{D}\left\langle b, S_{1}-a\right\rangle=\mathbf{D} F-\langle b, C b\rangle=\mathbf{D} F-\langle b, c\rangle,
$$

где $\mathbf{D}$ означает дисперсию. Очевидно, что если $\sigma^{2}=0$, то

$$
I_{\mathrm{NGD}(R)}(F)=I_{\mathrm{NGD}}(F)=I_{\mathrm{NA}}(F)=\left\{\left\langle b, S_{0}-a\right\rangle+\mathbf{E} F\right\} .
$$

Будем далее предполагать, что $\sigma^{2}>0$.

Очевидно, что $I_{\mathrm{NA}}(F)=\mathbf{R}$. 
Для того чтобы найти $I_{\mathrm{NGD}}(F)$, заметим, что $I_{\mathrm{NGD}}(F)=\left\langle b, S_{0}-a\right\rangle+$ $\mathbf{E} F+I_{\mathrm{NGD}}(\widetilde{F})$. Обозначим через $L$ образ $\mathbf{R}^{d}$ при отображении $x \mapsto C x$. Тогда корректно определено обратное отображение $C^{-1}: L \rightarrow L$. Поскольку $u$ инвариантна по распределению, то существует константа $\gamma>0$ такая, что для любой гауссовской случайной величины $\xi$ со средним $m$ и дисперсией $\sigma^{2}$ имеем $u(\xi)=m-\gamma \sigma$. Отсюда легко видеть, что множество $\widetilde{G}:=\left\{\mathbf{E}_{\mathbf{Q}}\left(S_{1}, \widetilde{F}\right): \mathbf{Q} \in \mathscr{R} \mathscr{D}\right\}$ имеет вид

$$
\widetilde{G}=(a, 0)+\left\{(x, y): x \in L, y \in \mathbf{R},\left\langle x, C^{-1} x\right\rangle+\sigma^{-2} y^{2} \leqslant \gamma^{2}\right\} .
$$

Следовательно,

$$
I_{\mathrm{NGD}}(F)=\left[\left\langle b, S_{0}-a\right\rangle+\mathbf{E} F-\alpha,\left\langle b, S_{0}-a\right\rangle+\mathbf{E} F+\alpha\right],
$$

где

$$
\alpha=\left(\sigma^{2} \gamma^{2}-\sigma^{2}\left\langle S_{0}-a, C^{-1}\left(S_{0}-a\right)\right\rangle\right)^{1 / 2} .
$$

(В частности, условие NGD первого рода выполнено тогда и только тогда, когда $\left\langle S_{0}-a, C^{-1}\left(S_{0}-a\right)\right\rangle \leqslant \gamma^{2}$.)

Аналогично проверяем, что

$$
I_{\mathrm{NGD}(R)}(F)=\left[\left\langle b, S_{0}-a\right\rangle+\mathbf{E} F-\alpha(R),\left\langle b, S_{0}-a\right\rangle+\mathbf{E} F+\alpha(R)\right],
$$

где $\alpha(R)=\left(\sigma^{2} \gamma^{2} R^{2} /(1+R)^{2}-\sigma^{2}\left\langle S_{0}-a, C^{-1}\left(S_{0}-a\right)\right\rangle\right)^{1 / 2}$. В частности, условие NGD второго рода выполнено тогда и только тогда, когда

$$
\left\langle S_{0}-a, C^{-1}\left(S_{0}-a\right)\right\rangle \leqslant \frac{\gamma^{2} R^{2}}{(1+R)^{2}} .
$$

Заметим, что $I_{\mathrm{NGD}}(F)$ и $I_{\mathrm{NGD}(R)}(F)$ зависят от $u$ весьма слабо, т.е. они зависят лишь от $\gamma$.

3.4. Динамическая модель с бесконечным числом активов. Пусть $\left(\Omega, \mathscr{F},\left(\mathscr{F}_{t}\right)_{t \in[0, T]}, \mathbf{P}\right)$ - фильтрованное вероятностное пространство. Предполагаем, что $\sigma$-алгебра $\mathscr{F}_{0}$ тривиальна. Пусть $\mathscr{D} \subseteq \mathscr{P}-$ выпуклое слабо компактное множество (в постановке п. $3.1 \mathscr{D}$ - определяющее множество $u$; в постановке п. $3.2 \mathscr{D}=(1+R)^{-1} \mathscr{P} \mathscr{D}+R(1+$ $\left.R)^{-1} \mathscr{R} \mathscr{D}\right)$. Пусть $\left(S^{i}\right), i \in I$, - семейство $\left(\mathscr{F}_{t}\right)$-согласованных непрерывных справа с пределами слева процессов (множество $I$ произвольно, и мы не налагаем никаких ограничений на вероятностную структуру $S^{i}$, как, например, предположение, что $S^{i}$ - семимартингал). С финансовой точки зрения, $S^{i}$ - дисконтированный процесс цены $i$-го актива. Мы предполагаем, что $S_{t}^{i} \in L_{\mathrm{s}}^{1}(\mathscr{D})$ для любых $t \in[0, T], i \in I$. Множество возможных прибылей, которые в этой модели можно получить при помощи кусочно-постоянных стратегий (а только такие стратегии 
и можно реализовать на практике), естественно определить следующим образом:

$$
\begin{gathered}
A=\left\{\sum_{n=1}^{N} \sum_{i \in I} H_{n}^{i}\left(S_{u_{n}}^{i}-S_{u_{n-1}}^{i}\right): N \in \mathbf{N},\right. \\
u_{0} \leqslant \cdots \leqslant u_{N}-\left(\mathscr{F}_{t}\right) \text {-моменты остановки, } \\
H_{n}^{i} \text { является } \mathscr{F}_{u_{n-1}} \text {-измеримой и } \\
\left.H_{n}^{i}=0 \text { для всех } i, \text { кроме конечного числа }\right\} .
\end{gathered}
$$

Следующая лемма показывает, что в данной модели множество $A$ $\mathscr{D}$-согласовано, а также дает явный вид множества $\mathscr{D} \cap \mathscr{R}$.

Лемма 3.1. Имеем $\mathscr{D} \cap \mathscr{R}=\mathscr{D} \cap \mathscr{R}\left(A^{\prime}\right)=\mathscr{D} \cap \mathscr{M}$, где

$$
\begin{aligned}
& A^{\prime}=\{ H\left(S_{v}^{i}-S_{u}^{i}\right): u \leqslant v \in[0, T], i \in I, \\
&\left.H \text { является } \mathscr{F}_{u} \text {-измеримой и ограниченной }\right\}, \\
& \mathscr{M}=\{\mathbf{Q} \in \mathscr{P}: \text { для любого } i \in I \\
&\left.\quad \text { прочесс } S^{i} \text { является }\left(\mathscr{F}_{t}, \mathbf{Q}\right) \text {-мартингалом }\right\} .
\end{aligned}
$$

Д о к а з а т е л ь с т в о. Включения $\mathscr{D} \cap \mathscr{R} \subseteq \mathscr{D} \cap \mathscr{R}\left(A^{\prime}\right) \subseteq \mathscr{D} \cap \mathscr{M}$ очевидны. Поэтому достаточно доказать включение $\mathscr{D} \cap \mathscr{M} \subseteq \mathscr{D} \cap \mathscr{R}$. Пусть $\mathrm{Q} \in \mathscr{D} \cap \mathscr{M}$. Возьмем $X=\sum_{n=1}^{N} \sum_{i \in I} H_{n}^{i}\left(S_{u_{n}}^{i}-S_{u_{n-1}}^{i}\right) \in A$. Процесс

$$
M_{k}=\sum_{n=1}^{k} \sum_{i \in I} H_{n}^{i}\left(S_{u_{n}}^{i}-S_{u_{n-1}}^{i}\right), \quad k=0,1, \ldots, N,
$$

является $\left(\mathscr{F}_{u_{k}}, \mathbf{Q}\right)$-локальным мартингалом. Предположим, что $\mathbf{E}_{\mathbf{Q}} X^{-}<$ $\infty$ (иначе $\left.\mathbf{E}_{\mathbf{Q}} X=-\infty\right)$. Тогда $M-$ мартингал (см. [64, гл. II, §1c]), так что $\mathbf{E}_{\mathbf{Q}} X=\mathbf{E}_{\mathbf{Q}} M_{N}=0$. Итак, в любом случае $\mathbf{E}_{\mathbf{Q}} X \leqslant 0$, что доказывает включение $\mathrm{Q} \in \mathscr{R}$. Лемма 3.1 доказана.

П р и м е p 3.4. Рассмотрим метод NGD второго рода в применении к модели Блэка-Шоулса-Мертона. Итак, $S_{t}=S_{0} e^{\mu t+\sigma B_{t}}$, $\mathscr{F}_{t}=\mathscr{F}_{t}^{B}$, где $B-$ броуновское движение; $\mathscr{P} \mathscr{D}=\{\mathbf{P}\}$ и задано множество $\mathscr{R} \mathscr{D}$, определяющее риск. Достаточно неожиданно, что в этой модели $\sup _{X \in A} \operatorname{RAROC}(X)=\infty$. Действительно, множество $\mathscr{M}$ состоит из единственной меры $\mathbf{Q}_{0}$, причем плотность $d \mathbf{Q}_{0} / d \mathbf{P}$ не отделена от нуля. Таким образом, условие (3.2) нарушается для любого $R>0$.

Построим явно последовательность $X_{n} \in A$, для которой $\operatorname{RAROC}\left(X_{n}\right) \rightarrow \infty$. Рассмотрим $D_{n}=\left\{d \mathbf{Q}_{0} / d \mathbf{P}<n^{-1}\right\}$ и положим $X_{n}=a_{n} I\left(D_{n}\right)-I\left(\Omega \backslash D_{n}\right)$, где $a_{n}$ выбрано так, что $\mathbf{E}_{\mathbf{Q}_{0}} X_{n}=0$. Тогда $\mathbf{E}_{\mathbf{P}} X_{n} \rightarrow \infty$, в то время как $\inf _{\mathbf{Q} \in \mathscr{R} \mathscr{D}} \mathbf{E}_{\mathbf{Q}} X \geqslant-1$, так что 
$\operatorname{RAROC}\left(X_{n}\right) \rightarrow \infty$. На самом деле, $X_{n} \notin A$, но для любого $n$ можно построить $\left(Y_{n}^{m}\right) \in A$ так, что $-2 \leqslant Y_{n}^{m} \leqslant a_{n}+1$ и $Y_{n}^{m} \longrightarrow \mathbf{P} X_{n}$ при $m \rightarrow \infty$. Тогда $\operatorname{RAROC}\left(Y_{n}^{m}\right) \longrightarrow \operatorname{RAROC}\left(X_{n}\right)$ при $m \rightarrow \infty$, так что $\operatorname{RAROC}\left(Y_{n}^{m(n)}\right) \rightarrow \infty$ для некоторой подпоследовательности $m(n)$.

Этот пример показывает, что метод NGD второго рода обычно несовместим с полными моделями. Но этот метод нацелен как раз на неполные модели, поскольку в полных уже метод арбитража дает точную цену .

Отметим также, что условие NGD первого рода может выполняться в модели Блэка-Шоулса-Мертона при подходящем выборе когерентной функции полезности $u$.

3.5. Динамическая модель с операционными издержками. Пусть $\left(\Omega, \mathscr{F},\left(\mathscr{F}_{t}\right)_{t \in[0, T]}, \mathbf{P}\right)$ - фильтрованное вероятностное пространство. Предполагаем, что $\sigma$-алгебра $\mathscr{F}_{0}$ тривиальна и фильтрация $\left(\mathscr{F}_{t}\right)$ непрерывна справа. Пусть $\mathscr{D} \subseteq \mathscr{P}$ - выпуклое слабо компактное множество. Пусть $S^{a i}, S^{b i}, i \in I$, - два семейства $\left(\mathscr{F}_{t}\right)$-согласованных непрерывных справа с пределами слева процессов. С финансовой точки зрения, $S^{a i}$ (соответственно $S^{b i}$ ) - дисконтированный процесс цены продавца (соответственно покупателя) для $i$-го актива, так что $S^{a} \geqslant S^{b}$ покомпонентно. Предполагаем, что $S_{t}^{a i}, S_{t}^{b i} \in L_{\mathrm{s}}^{1}(\mathscr{D})$ для любых $t \in[0, T]$, $i \in I$. Множество возможных прибылей в этой модели естественно определить следующим образом:

$$
\begin{gathered}
A=\left\{\sum_{n=0}^{N} \sum_{i \in I}\left[-H_{n}^{i} I\left(H_{n}^{i}>0\right) S_{u_{n}}^{a i}-H_{n}^{i} I\left(H_{n}^{i}<0\right) S_{u_{n}}^{b i}\right]:\right. \\
N \in \mathbf{N}, u_{0} \leqslant \cdots \leqslant u_{N}-\left(\mathscr{F}_{t}\right) \text {-моменты остановки, } \\
H_{n}^{i} \text { является } \mathscr{F}_{u_{n}} \text {-измеримой, } \\
H_{n}^{i}=0 \text { для всех } i, \text { кроме конечного числа, } \\
\text { и } \left.\sum_{n=0}^{N} H_{n}^{i}=0 \text { для любого } i\right\} .
\end{gathered}
$$

Здесь $H_{n}^{i}$ означает количество единиц $i$-го актива, которое покупаем в момент $u_{n}$ (так что $\sum_{k=0}^{n} H_{k}^{i}$ - общее количество единиц $i$-го актива, имеющееся в момент $\left.u_{n}\right)$. Заметим, что при отсутствии операционных издержек (т.е. если $S^{a i}=S^{b i}=S^{i}$ для любого $i$ ) множество $A$ совпадает с множеством, заданным по формуле (3.4).

Следующая лемма показывает, что в данной модели $A \mathscr{D}$-согласовано, а также дает явный вид множества $\mathscr{D} \cap \mathscr{R}$. Напомним, что момент остановки называется простым, если он принимает лишь конечное число значений. 
Лемма 3.2. Имеем $\mathscr{D} \cap \mathscr{R}=\mathscr{D} \cap \mathscr{R}\left(A^{\prime}\right)=\mathscr{D} \cap \mathscr{M}$, где

$$
\begin{aligned}
& A^{\prime}=\left\{G\left(S_{v}^{b i}-S_{u}^{a i}\right)+H\left(-S_{v}^{a i}+S_{u}^{b i}\right): i \in I, u \leqslant v-\right. \\
& \text { простьие }\left(\mathscr{F}_{t}\right) \text {-моменты остановки, а }
\end{aligned}
$$

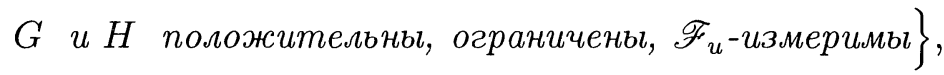

$$
\begin{aligned}
& \mathscr{M}=\left\{\mathbf{Q} \in \mathscr{P}: \text { для любого } і \text { существует }\left(\mathscr{F}_{t}, \mathbf{Q}\right) \text {-мартингал } M^{i}\right. \\
& \text { mакой, что } \left.S^{b i} \leqslant M^{i} \leqslant S^{a i}\right\} \text {. }
\end{aligned}
$$

Д о к а з а т е л ь с т в о. Включение $\mathscr{D} \cap \mathscr{R} \subseteq \mathscr{D} \cap \mathscr{R}\left(A^{\prime}\right)$ очевидно.

Докажем включение $\mathscr{D} \cap \mathscr{R}\left(A^{\prime}\right) \subseteq \mathscr{D} \cap \mathscr{M}$. Возьмем $\mathrm{Q} \in \mathscr{D} \cap \mathscr{R}\left(A^{\prime}\right)$. Фиксируем $i \in I$. Для любых простых моментов остановки $u \leqslant v$ имеем $S_{u}^{a i}, S_{u}^{b i}, S_{v}^{a i}, S_{v}^{b i} \in L_{\mathrm{s}}^{1}(\mathscr{D})$ и

$$
\mathbf{E}_{\mathbf{Q}}\left(S_{v}^{a i} \mid \mathscr{F}_{u}\right) \geqslant S_{u}^{b i}, \quad \mathbf{E}_{\mathbf{Q}}\left(S_{v}^{b i} \mid \mathscr{F}_{u}\right) \leqslant S_{u}^{a i} .
$$

Рассмотрим огибающие Снелла

$$
\begin{array}{cc}
X_{t}=\underset{\tau \in \mathcal{T}_{t}}{\operatorname{ess} \sup } \mathbf{E}_{\mathbf{Q}}\left(S_{\tau}^{b i} \mid \mathscr{F}_{t}\right), & t \in[0, T], \\
Y_{t}=\underset{\tau \in \mathcal{T}_{t}}{\operatorname{essinf}} \mathbf{E}_{\mathbf{Q}}\left(S_{\tau}^{a i} \mid \mathscr{F}_{t}\right), & t \in[0, T],
\end{array}
$$

где $\mathcal{T}_{t}$ - множество простых $\left(\mathscr{F}_{t}\right)$-моментов остановки таких, что $\tau \geqslant t$. (Напомним, что $\operatorname{ess~sup}_{\alpha} \xi_{\alpha}$ - такая случайная величина $\xi$, что для любого $\alpha$ выполнено $\xi \geqslant \xi_{\alpha}$ п.н. и для любой другой случайной величины $\xi^{\prime}$ с этим свойством выполнено $\xi \leqslant \xi^{\prime}$ п.н.) Тогда $X$ является $\left(\mathscr{F}_{t}\right)$-супермартингалом, а $Y$ является $\left(\mathscr{F}_{t}, \mathbf{Q}\right)$-субмартингалом (см. [30, теорема 2.12.1]).

Докажем, что для любого $t \in[0, T]$ выполнено неравенство $X_{t} \leqslant Y_{t}$ $\mathrm{Q}$-п.н. Допустим, что существует $t$ такое, что $\mathbf{P}\left(X_{t}>Y_{t}\right)>0$. Тогда найдутся $\tau, \sigma \in \mathcal{T}_{t}$ такие, что

$$
\mathbf{Q}\left(\mathbf{E}_{\mathbf{Q}}\left(S_{\tau}^{b i} \mid \mathscr{F}_{t}\right)>\mathbf{E}_{\mathbf{Q}}\left(S_{\sigma}^{a i} \mid \mathscr{F}_{t}\right)\right)>0 .
$$

Отсюда следует, что $\mathbf{Q}(\xi>\eta)>0$, где $\xi=\mathbf{E}_{\mathbf{Q}}\left(S_{\tau}^{b i} \mid \mathscr{F}_{\tau \wedge \sigma}\right)$ и $\eta=$ $\mathbf{E}_{\mathbf{Q}}\left(S_{\sigma}^{a i} \mid \mathscr{F}_{\tau \wedge \sigma}\right)$. Предположим сначала, что $\mathbf{Q}(\{\xi>\eta\} \cap\{\tau \leqslant \sigma\})>0$. На множестве $\{\tau \leqslant \sigma\}$ имеем

$$
\xi=S_{\tau}^{b i}=S_{\tau \wedge \sigma}^{b i}, \quad \eta=\mathbf{E}_{\mathbf{Q}}\left(S_{\sigma}^{a i} \mid \mathscr{F}_{\tau \wedge \sigma}\right)=\mathbf{E}_{\mathbf{Q}}\left(S_{\tau \vee \sigma}^{a i} \mid \mathscr{F}_{\tau \wedge \sigma}\right)
$$

и получаем противоречие с (3.5). Аналогично приходим к противоречию, если предположить, что $\mathrm{Q}(\{\xi>\eta\} \cap\{\tau \geqslant \sigma\})>0$. В результате $X_{t} \leqslant Y_{t}$ Q-п.н. Из [42, лемма 3] теперь следует, что сушествует $\left(\mathscr{F}_{t}, \mathrm{Q}\right)$-мартингал $M$ такой, что $X \leqslant M \leqslant Y$. Итак, $\mathrm{Q} \in \mathscr{M}$. 
Докажем включение $\mathscr{D} \cap \mathscr{M} \subseteq \mathscr{D} \cap \mathscr{R}$. Возьмем $\mathrm{Q} \in \mathscr{D} \cap \mathscr{M}$, так что для каждого $i$ существует $\left(\mathscr{F}_{t}, \mathrm{Q}\right)$-мартингал $M^{i}$ такой, что $S^{b i} \leqslant$ $M^{i} \leqslant S^{a i}$. Для любого

$$
X=\sum_{n=0}^{N} \sum_{i \in I}\left[-H_{n}^{i} I\left(H_{n}^{i}>0\right) S_{u_{n}}^{a i}-H_{n}^{i} I\left(H_{n}^{i}<0\right) S_{u_{n}}^{b i}\right] \in A
$$

имеем

$$
\begin{aligned}
X & \leqslant \sum_{n=0}^{N} \sum_{i \in I}\left[-H_{n}^{i} I\left(H_{n}^{i}>0\right) M_{u_{n}}^{i}-H_{n}^{i} I\left(H_{n}^{i}<0\right) M_{u_{n}}^{i}\right] \\
& =\sum_{n=1}^{N} \sum_{i \in I}\left(\sum_{k=0}^{n-1} H_{k}^{i}\right)\left(M_{u_{n}}^{i}-M_{u_{n-1}}^{i}\right) .
\end{aligned}
$$

Повторяя рассуждения из доказательства леммы 3.1 , получаем $\mathbf{E}_{\mathbf{Q}} X \leqslant 0$. Итак, $\mathbf{Q} \in \mathscr{R}$. Лемма 3.2 доказана.

Рассмотрим теперь модель с пропорциональными операционными издержками, т.е. $S^{a i}=S^{i}, S^{b i}=\left(1-\lambda^{i}\right) S^{i}$, где каждый процесс $S^{i}$ положителен, а $\lambda^{i} \in(0,1)$. Обозначим интервал справедливых NGD-цен в этой модели через $I_{\lambda}(F)$ (можно рассматривать метод NGD как первого, так и второго рода, поскольку последний сводится к первому путем рассмотрения $\left.\mathscr{D}=(1+R)^{-1} \mathscr{P} \mathscr{D}+R(1+R)^{-1} \mathscr{R} \mathscr{D}\right)$. Пусть $\left(\lambda_{n}\right)=\left(\lambda_{n} ; i \in I, n \in \mathbf{N}\right)$ - последовательность такая, что $\lambda_{n}^{i} \rightarrow 0$ при $n \rightarrow \infty$ для любого $i$.

Теорема 3.2. Для $F \in L_{\mathbf{s}}^{1}(\mathscr{D})$ справедливо соотношение $I_{\lambda_{n}}(F) \rightarrow$ $I_{0}(F)$ при $n \rightarrow \infty$ в том смиссле, ито правые (соответственно левые) кончевые точки $I_{\lambda_{n}}(F)$ сходятся $\kappa$ правой (соответственно левой) концевой точке $I_{0}(F)$.

Д о к а з а т е л ь с т в о. Обозначим $r$ правую концевую точку интервала $I_{0}(F)$. Допустим, что правые концевые точки $I_{\lambda_{n}}(F)$ не сходятся к $r$. Поскольку $I_{0}(F) \subseteq I_{\lambda_{n}}(F)$, это означает, что существует $r^{\prime}>r$ такое, что для любого $n$ (возможно, после перехода к подпоследовательности) найдется $\mathbf{Q}_{n} \in \mathscr{D} \cap \mathscr{R}_{\lambda_{n}}$ со свойством: $\mathbf{E}_{\mathbf{Q}} F \geqslant r^{\prime}\left(\mathscr{R}_{\lambda}-\right.$ множество риск-нейтральных мер в модели с коэффициентом операционных издержек $\lambda)$. Последовательность $\left(\mathbf{Q}_{n}\right)$ обладает слабой предельной точкой $\mathbf{Q}_{\infty} \in \mathscr{D}$. Фиксируем $i \in I, u \leqslant v \in[0, T]$ и положительную ограниченную $\mathscr{F}_{u}$-измеримую случайную величину $H$. Для любого $n$ имеем $\mathbf{E}_{\mathbf{Q}_{n}} H\left(\left(1-\lambda_{n}^{i}\right) S_{v}^{i}-S_{u}^{i}\right) \leqslant 0$. Поскольку $S_{v}^{i} \in L_{\mathbf{s}}^{1}(\mathscr{D})$, то $\sup _{\mathbf{Q} \in \mathscr{D}} \mathbf{E}_{\mathbf{Q}} S_{v}^{i}<\infty$, и поэтому $\lim \sup _{n} \mathbf{E}_{\mathbf{Q}_{n}} H\left(S_{v}^{i}-S_{u}^{i}\right) \leqslant 0$. Поскольку отображение $\mathscr{D} \ni \mathbf{Q} \mapsto \mathbf{E}_{\mathbf{Q}} H\left(S_{v}^{i}-S_{u}^{i}\right)$ слабо непрерывно, получаем $\mathbf{E}_{\mathbf{Q}_{\infty}} H\left(S_{v}^{i}-S_{u}^{i}\right) \leqslant 0$. Аналогично доказывается, что $\mathbf{E}_{\mathbf{Q}_{\infty}} H\left(-S_{v}^{i}+S_{u}^{i}\right) \leqslant 0$. Итак, $S^{i}$ является $\left(\mathscr{F}_{t}, \mathbf{Q}_{\infty}\right)$-мартингалом, так что $\mathbf{Q}_{\infty} \in \mathscr{D} \cap \mathscr{R}_{0}$. Поскольку отображение $\mathscr{D} \ni \mathbf{Q} \mapsto \mathbf{E}_{\mathbf{Q}} F$ слабо непрерывно, то $\mathbf{E}_{\mathbf{Q}_{\infty}} F \geqslant r^{\prime}$, что является противоречием. Теорема 3.2 доказана. 
3.6. Хеджирование. Рассмотрим модель п. 3.1.

О п р е д е л е н и е 3.8. Верхняя и нижняя $N G D$-цены платежного поручения $F$ определяются следующим образом:

$$
\begin{aligned}
& \bar{V}(F)=\inf \{x: \text { существует } X \in A \text { такой, что } u(X-F+x) \geqslant 0\}, \\
& \underline{V}(F)=\sup \{x: \text { существует } X \in A \text { такой, что } u(X+F-x) \geqslant 0\} .
\end{aligned}
$$

Проблема нахождения $\bar{V}(F)$ имеет сходство с проблемой суперхеджирования, рассмотренной Цвитаничем и Каратзасом [18], а также Секине [63], но отличие состоит в том, что в этих работах риск измеряется не как $\rho(X-F+x)$, а как $\rho\left((X-F+x)^{-}\right)$.

Предложение 3.1. Если $A-$ конус, а $F \in L_{\mathrm{s}}^{1}(\mathscr{D})$, mo

$$
\bar{V}(F)=\sup \left\{\mathbf{E}_{\mathbf{Q}} F: \mathbf{Q} \in \mathscr{D} \cap \mathscr{R}\right\}, \quad \underline{V}(F)=\inf \left\{\mathbf{E}_{\mathbf{Q}} F: \mathbf{Q} \in \mathscr{D} \cap \mathscr{R}\right\} .
$$

Док аз а те ль с т в о. Возьмем $x_{0} \in \mathbf{R}$ и положим $A\left(x_{0}\right)=A+$ $\left\{h\left(x_{0}-F\right): h \in \mathbf{R}_{+}\right\}$. Используя теорему 3.1 , можем написать

$$
\begin{aligned}
\bar{V}(F) \geqslant x_{0} & \Longleftrightarrow \text { не существует } X \in A \text { такого, что } u\left(X-F+x_{0}\right)>0 \\
& \Longleftrightarrow \text { не существует } X \in A\left(x_{0}\right) \text { такого, что } u(X)>0 \\
& \Longleftrightarrow \mathscr{D} \cap \mathscr{R}\left(A\left(x_{0}\right)\right) \neq \varnothing \\
& \Longleftrightarrow \text { существует } \mathbf{Q} \in \mathscr{D} \cap \mathscr{R} \text { такая, что } \mathbf{E}_{\mathbf{Q}} F \geqslant x_{0} .
\end{aligned}
$$

Это доказывает формулу для $\bar{V}(F)$. Представление для $\underline{V}(F)$ доказывается аналогично. Предложение 3.1 доказано.

3 а м е ч а н и я. (i) Формально утверждение верно и при нарушении условия NGD. В этом случае $\bar{V}(F)=-\infty$ и $\underline{V}(F)=\infty$.

(ii) Из доказательства видно, что существуют $\overline{\mathbf{Q}}, \underline{\mathbf{Q}} \in \mathscr{D} \cap \mathscr{R}$ такие, что $\mathbf{E}_{\overline{\mathbf{Q}}} F=\bar{V}(F), \mathbf{E}_{\underline{\mathbf{Q}}}(F)=\underline{V}(F)$. Этим метод NGD отличается от метода отсутствия арбитража.

(iii) При выполнении условий предложения выполнено равенство $I_{\mathrm{NGD}}(F)=[\underline{V}(F), \bar{V}(F)]$.

Рассмотрим теперь проблему супер- и субхеджирования для случая статической модели с конечным числом активов без операционных издержек. Итак, заданы выпуклое множество $\mathscr{D} \subseteq \mathscr{P}, S_{0} \in \mathbf{R}^{d}$ и $S_{1}^{1}, \ldots, S_{1}^{d} \in L_{\mathrm{w}}^{1}(\mathscr{D})$. С финансовой точки зрения, $S_{n}^{i}$ - дисконтированная цена $i$-го актива в момент $n$.

О п р е д е л е н и е 3.9. Суперхеджирующая и субхеджирующая стратегии определяются следующим образом:

$$
\begin{aligned}
& \bar{H}(F)=\left\{h \in \mathbf{R}^{d}: u\left(\left\langle h, S_{1}-S_{0}\right\rangle-F+\bar{V}(F)\right) \geqslant 0\right\}, \\
& \underline{H}(F)=\left\{h \in \mathbf{R}^{d}: u\left(\left\langle h, S_{1}-S_{0}\right\rangle+F-\underline{V}(F)\right) \geqslant 0\right\} .
\end{aligned}
$$


Ниже мы даем простое геометрическое описание этих стратегий. Предположим, что $F \in L_{\mathrm{w}}^{1}(\mathscr{D})$, и введем обозначения

$$
\begin{aligned}
G & =\operatorname{cl}\left\{\mathbf{E}_{\mathbf{Q}}\left(S_{1}, F\right): \mathbf{Q} \in \mathscr{D}\right\}, \\
\bar{v} & =\sup \left\{x:\left(S_{0}, x\right) \in G\right\}, \quad \underline{v}=\inf \left\{x:\left(S_{0}, x\right) \in G\right\}, \\
\bar{N} & =\left\{h \in \mathbf{R}^{d+1}: \forall x \in G\left\langle h, x-\left(S_{0}, \bar{v}\right)\right\rangle \geqslant 0\right\}, \\
\underline{N} & =\left\{h \in \mathbf{R}^{d+1}: \forall x \in G\left\langle h, x-\left(S_{0}, \underline{v}\right)\right\rangle \geqslant 0\right\},
\end{aligned}
$$

т.е. $G$ - генератор для $\left(S_{1}, F\right)$ и $u ; \bar{N}$ (соответственно $\left.\underline{N}\right)-$ множество внутренних нормалей к $G$ в точке $\left(S_{0}, \bar{v}\right)$ (соответственно $\left(S_{0}, \underline{v}\right)$ ); см. рис. 4.

\section{Предложение 3.2. Имеем}

$$
\begin{gathered}
\bar{V}(F)=\bar{v}, \quad \underline{V}(F)=\underline{v}, \\
\bar{H}=\left\{h \in \bar{N}: h^{d+1}=-1\right\}, \quad \underline{H}=\left\{h \in \underline{N}: h^{d+1}=1\right\} .
\end{gathered}
$$

3 а м е ч а н и е. Утверждение формально верно и при нарушении условия NGD. В этом случае $S_{0}$ не принадлежит проекции $G$ на $\mathbf{R}^{d}$, $\bar{v}=\bar{V}(F)=-\infty, \underline{v}=\underline{V}(F)=\infty, \bar{N}=\bar{H}=\varnothing, \underline{N}=\underline{H}=\varnothing$.

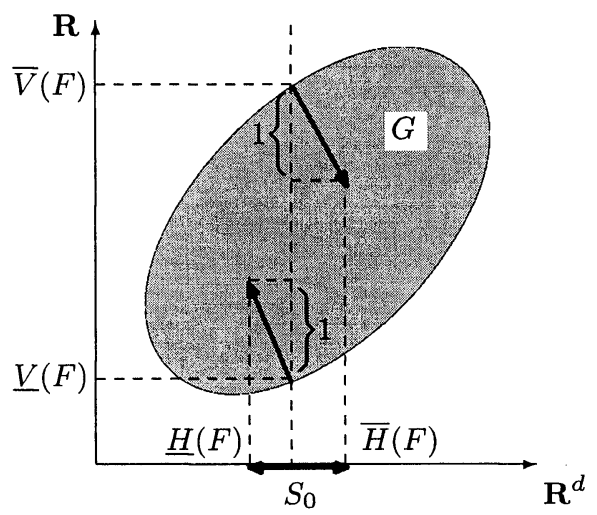

Рис. 4. Решение задачи супер- и субхеджирования

Д ок а з а т ел ь с т в о п ре д ло жен и я 3.2. Докажем утверждение для $\underline{V}(F)$ и $\underline{H}$. Легко видеть, что $\underline{V}(F)=\sup _{h} u\left(\left\langle h, S_{1}-S_{0}\right\rangle+F\right)$ и $\underline{H}=\arg \max _{h} u\left(\left\langle h, S_{1}-S_{0}\right\rangle+F\right)$. Имеем

$$
\begin{aligned}
u\left(\left\langle h, S_{1}-S_{0}\right\rangle+F\right) & =\inf _{z \in G}\left\langle(h, 1), z-\left(S_{0}, 0\right)\right\rangle \\
& \leqslant\left\langle(h, 1),\left(S_{0}, \underline{v}\right)-\left(S_{0}, 0\right)\right\rangle=\underline{v}, \quad h \in \mathbf{R}^{d} .
\end{aligned}
$$


С другой стороны, при $h \in \underline{H}$ (и только при таких $h$ ) неравенство выше преврашается в равенство. Предложение 3.2 доказано.

П р и м е р 3.5. Вернемся к примеру 3.3. Из результатов этого примера видно, что

$$
\bar{V}(F)=\left\langle b, S_{0}-a\right\rangle+\mathbf{E} F+\alpha, \quad \underline{V}(F)=\left\langle b, S_{0}-a\right\rangle+\mathbf{E} F-\alpha,
$$

где $\alpha=\left(\sigma^{2} \gamma^{2}-\sigma^{2}\left\langle S_{0}-a, C^{-1}\left(S_{0}-a\right)\right\rangle\right)^{1 / 2}$. Для нахождения $\bar{H}$ и $\underline{H}$ выразим верхнюю и нижнюю границы множества $\widetilde{G}$, заданного формулой (3.3), как $y= \pm\left(\sigma^{2} \gamma^{2}-\sigma^{2}\left\langle x-a, C^{-1}(x-a)\right\rangle\right)^{1 / 2}$. Дифференцированием получаем

$$
\begin{aligned}
\bar{H}(\widetilde{F}) & =\underline{H}(\widetilde{F})=\left.d\right|_{x=S_{0}}\left(\sigma^{2} \gamma^{2}-\sigma^{2}\left\langle x-a, C^{-1}(x-a)\right\rangle\right)^{1 / 2} \\
& =-\sigma^{2} \alpha^{-1} C^{-1}\left(S_{0}-a\right) .
\end{aligned}
$$

Следовательно,

$$
\bar{H}(F)=b-\sigma^{2} \alpha^{-1} C^{-1}\left(S_{0}-a\right), \quad \underline{H}(F)=-b-\sigma^{2} \alpha^{-1} C^{-1}\left(S_{0}-a\right) .
$$

Рассмотрим еще один важный пример. Пусть $S_{0} \in(0, \infty)$, а $S_{1}$ интегрируемая случайная величина такая, что Law $S_{1}$ не имеет атомов и supp Law $S_{1}=\mathbf{R}_{+}$. Пусть $u$ - когерентная функция полезности, отвечающая хвостовому V@R порядка $\lambda \in(0,1]$ (см. пример 2.1 (i)). Предполагаем, что $u\left(S_{1}\right)<S_{0}<-u\left(-S_{1}\right)$. Наконец, пусть $F=f\left(S_{1}\right)$, где $f: \mathbf{R}_{+} \rightarrow \mathbf{R}-$ выпуклая функция линейного роста. Обозначим Law $S_{1}$ через $\mathbf{Q}$ и найдем $a, b, c, d$ такие, что $a+b=\lambda, d-c=\lambda$ и

$$
\lambda^{-1} \int_{0}^{q_{a}} x \mathbf{Q}(d x)+\lambda^{-1} \int_{q_{1-b}}^{\infty} x \mathbf{Q}(d x)=S_{0}, \quad \lambda^{-1} \int_{q_{c}}^{q_{d}} x \mathbf{Q}(d x)=S_{0},
$$

где $q_{x}-x$-квантиль меры $\mathbf{Q}$.

Предложение 3.3. Имеем

$$
\begin{aligned}
& \bar{V}(F)=\lambda^{-1} \int_{0}^{q_{a}} f(x) \mathbf{Q}(d x)+\lambda^{-1} \int_{q_{1-b}}^{\infty} f(x) \mathbf{Q}(d x), \\
& \underline{V}(F)=\lambda^{-1} \int_{q_{c}}^{q_{d}} f(x) \mathbf{Q}(d x), \\
& \bar{H}(F)=\frac{f\left(q_{1-b}\right)-f\left(q_{a}\right)}{q_{1-b}-q_{a}}, \quad \underline{H}(F)=-\frac{f\left(q_{d}\right)-f\left(q_{c}\right)}{q_{d}-q_{c}} .
\end{aligned}
$$

Д о к а з а т ел ь с т о. Докажем сначала представление для $\bar{V}(F)$ при дополнительном предположении строгой выпуклости $f$. Согласно предложению 3.2 ,

$$
\bar{V}(F)=\sup _{Z \in \mathscr{D}_{\lambda}: \mathbf{E} Z X=S_{0}} \mathbf{E} Z f(X)
$$

$\left(\mathscr{D}_{\lambda}\right.$ задано формулой $\left.(2.3)\right)$. Найдем

$$
Z_{0} \in \underset{Z \in \mathscr{D}_{\lambda}: \mathbf{E} Z X=S_{0}}{\arg \max } \mathbf{E} Z f(X)
$$


(существование $Z_{0}$ следует из свойств компактности). Переходя от $Z_{0} \mathrm{~K}$ $\mathbf{E}\left(Z_{0} \mid X\right)$, можем предполагать, что $Z_{0}$ измерима относительно $X$, т.е. $Z_{0}=\varphi(X)$. Докажем, что

$$
Z_{0}=\lambda^{-1} I\left(X<q_{a}\right)+\lambda^{-1} I\left(X>q_{1-b}\right) .
$$

Допустим противное. Тогда найдутся $0<\alpha_{1}<\alpha_{2}<\alpha_{3}<\alpha_{4}$ такие, что $\mathbf{Q}\left(\left\{\varphi<\lambda^{-1}\right\} \cap\left(\alpha_{1}, \alpha_{2}\right)\right)>0, \mathbf{Q}\left(\{\varphi>0\} \cap\left(\alpha_{2}, \alpha_{3}\right)\right)>0, \mathbf{Q}(\{\varphi<$ $\left.\left.\lambda^{-1}\right\} \cap\left(\alpha_{3}, \alpha_{4}\right)\right)>0$. Для $h_{1}, h_{2}, h_{3} \in\left[0, \lambda^{-1}\right]$ положим

$$
\tilde{\varphi}(x)= \begin{cases}\varphi(x), & x \notin\left(\alpha_{1}, \alpha_{4}\right], \\ \varphi(x) \vee h_{1}, & x \in\left(\alpha_{1}, \alpha_{2}\right], \\ \varphi(x) \wedge h_{2}, & x \in\left(\alpha_{2}, \alpha_{3}\right], \\ \varphi(x) \vee h_{3}, & x \in\left(\alpha_{3}, \alpha_{4}\right] .\end{cases}
$$

Можно найти $h_{1}, h_{2}, h_{3}$ такие, что $\mathbf{Q}\left(\{\tilde{\varphi}>\varphi\} \cap\left(\alpha_{1}, \alpha_{2}\right)\right)>0, \mathbf{Q}(\{\tilde{\varphi}<$ $\left.\varphi\} \cap\left(\alpha_{2}, \alpha_{3}\right)\right)>0, \mathbf{Q}\left(\{\widetilde{\varphi}>\varphi\} \cap\left(\alpha_{3}, \alpha_{4}\right)\right)>0$,

$$
\begin{gathered}
\int_{0}^{\infty} x \widetilde{\varphi}(x) \mathbf{Q}(d x)=\int_{0}^{\infty} x \varphi(x) \mathbf{Q}(d x)=S_{0}, \\
\int_{0}^{\infty} \tilde{\varphi}(x) \mathbf{Q}(d x)=\int_{0}^{\infty} \varphi(x) \mathbf{Q}(d x)=1 .
\end{gathered}
$$

Рассмотрим аффинную функцию $\tilde{f}$, совпадающую с $f$ в точках $\alpha_{2}$ и $\alpha_{3}$. Тогда

$$
\int_{0}^{\infty}(\widetilde{\varphi}(x)-\varphi(x)) \tilde{f}(x) \mathbf{Q}(d x)=0 .
$$

Поскольку $f$ строго выпукла, то $\tilde{f}<f$ на $\left(\alpha_{1}, \alpha_{2}\right), \tilde{f}>f$ на $\left(\alpha_{2}, \alpha_{3}\right)$ и $\tilde{f}>f$ на $\left(\alpha_{3}, \alpha_{4}\right)$. Следовательно,

$$
\int_{0}^{\infty}(\widetilde{\varphi}(x)-\varphi(x)) f(x) \mathbf{Q}(d x)>0 .
$$

Итак, мы нашли плотность $\widetilde{Z}_{0}=\widetilde{\varphi}(X) \in \mathscr{D}_{\lambda}$ такую, что $\mathbf{E} \widetilde{Z}_{0} X=S_{0}$ и $\mathbf{E} \widetilde{Z}_{0} f(X)>\mathbf{E} Z_{0} f(X)$. Это противоречит выбору $Z_{0}$. В результате (3.6) выполнено, что доказывает требуемое представление $\bar{V}(F)$.

Теперь докажем представление для $\bar{V}(F)$ в общем случае. Рассмотрим $Z_{0}$, определенную формулой (3.6). Выберем строго выпуклую функцию $\tilde{f}$ линейного роста. Тогда функции $f_{\varepsilon}=f+\varepsilon \tilde{f}$ строго выпуклы и из доказанного выше результата следует, что $\mathbf{E} Z f_{\varepsilon}(X) \leqslant \mathbf{E} Z_{0} f_{\varepsilon}(X)$ для любого $Z \in \mathscr{D}_{\lambda}$. Переходя к пределу при $\varepsilon \downarrow 0$, получаем $\mathbf{E} Z f(X) \leqslant$ $\mathbf{E} Z_{0} f(X)$ для любого $Z \in \mathscr{D}_{\lambda}$. Это доказывает требуемое представление для $\bar{V}(F)$.

Докажем теперь представление для $\bar{H}(F)$. Рассмотрим функцию

$$
g(x)=\sup _{Z \in \mathscr{D}_{\lambda}: \mathbf{E} Z X=x} \mathbf{E} Z f(X), \quad x \in\left[u\left(S_{1}\right),-u\left(-S_{1}\right)\right] .
$$


Из рассуждений выше следует, что $g=g_{1} \circ g_{2}^{-1}$, где

$$
\begin{aligned}
& g_{1}(x)=\lambda^{-1} \int_{0}^{q_{x}} f(y) \mathbf{Q}(d y)+\lambda^{-1} \int_{q_{1-\lambda+x}}^{\infty} f(y) \mathbf{Q}(d y), \quad x \in\left[0, \lambda^{-1}\right], \\
& g_{2}(x)=\lambda^{-1} \int_{0}^{q_{x}} y \mathbf{Q}(d y)+\lambda^{-1} \int_{q_{1-\lambda+x}}^{\infty} y \mathbf{Q}(d y), \quad x \in\left[0, \lambda^{-1}\right] .
\end{aligned}
$$

Применяя предложение 3.2 , получаем

$$
\bar{H}(F)=g^{\prime}\left(S_{0}\right)=\frac{f\left(q_{1-b}\right)-f\left(q_{a}\right)}{q_{1-b}-q_{a}} .
$$

Представления для $\underline{V}(F)$ и $\underline{H}(F)$ доказываются аналогично. Предложение 3.3 доказано.

\section{СПИСОК ЛИТЕРАТУРЫ}

1. Acerbi $C$. Spectral measures of risk: a coherent representation of subjective risk aversion. - J. Banking and Finance, 2002, v. 26, p. 1505-1518.

2. Acerbi $C$. Coherent representations of subjective risk aversion. - Risk Measures for the 21st Century. Ed. by G. Szegö. New York: Wiley, 2004, p. 147-207.

3. Acerbi C., Tasche D. On the coherence of expected shortfall. - J. Banking and Finance, 2002, v. 26, № 7, p. 1487-1503.

4. Artzner P., Delbaen F., Eber J.-M., Heath D. Thinking coherently. - Risk, 1997, v. 10 , № 11, p. 68-71.

5. Artzner P., Delbaen F., Eber J.-M., Heath D. Coherent measures of risk. - Math. Finance, 1999, v. 9, № 3, p. 203-228.

6. Bernardo A., Ledoit O. Gain, loss, and asset pricing. - J. Political Economy, 2000, v. 108, № 1, p. 144-172.

7. Björk T., Slinko I. Towards a general theory of good deal bounds. www. newton.cam.ac.uk/webseminars/pg+ws/2005/dqf, Individual presentations, 25.01.2005.

8. Carlier G., Dana R.A. Core of convex distortions of a probability. - J. Economic Theory, 2003, v. 113, № 2, p. 199-222.

9. Carr P., Geman H., Madan D. Pricing and hedging in incomplete markets. J. Financial Economics, 2001, v. 62, p. 131-167.

10. Carr P., Geman H., Madan D. Pricing in incomplete markets: from absence of good deals to acceptable risk. - Risk Measures for the 21st Century. Ed. by G. Szegö. Wiley, 2004, p. 451-474.

11. Cerny A., Hodges S. The theory of good-deal pricing in financial markets. Mathematical Finance - Bachelier Congress (Paris, 2000). Ed. by H. Geman et al. Berlin: Springer, 2001, p. 175-202.

12. Cheridito P., Delbaen F., Kupper M. Dynamic monetary risk measures for bounded discrete-time processes. http://arxiv.org: math./0410453.

13. Cherny A.S. General arbitrage pricing model: probability approach. http://mech.math.msu.su/ ${ }^{\sim}$ cherny; Lecture Notes in Math. (to appear).

14. Cherny A.S. General arbitrage pricing model: transaction costs. http://mech.math.msu.su/ cherny; Lecture Notes in Math. (to appear).

15. Черный A.C. Равновесие на основе когерентных мер риска. http://mech.math.msu.su/ cherny.

16. Cherny A.S. Weighted V@R and its properties. - Finance Stoch., 2006, v. 10, № 2, p. 367-393.

17. Cochrane J.H., Saá-Requejo J. Beyond arbitrage: good-deal asset price bounds in incomplete markets. - J. Political Economy, 2000, v. 108, № 1, p. 79-119. 
18. Cvitanić J., Karatzas I. On dynamic measures of risk. - Finance Stoch., 1999, v. 3, № 4, p. 451-482.

19. Cvitanić J., Pham H., Touzi N. A closed-form solution to the problem of superreplication under transaction costs. - Finance Stoch., 1999, v. 3, № 1, p. 35-54.

20. Dalang R.C., Morton A., Willinger W. Equivalent martingale measures and noarbitrage in stochastic securities market models. - Stochastics Stochastics Rep., 1990, v. 29 , № 2, p. 185-201.

21. Delbaen F. Coherent risk measures on general probability spaces. Advances in Finance Stochastics. Essays in Honor of Dieter Sondermann. Ed. by K. Sandmann, P. Schönbucher. Berlin: Springer, 2002, p. 1-37.

22. Delbaen F. Coherent monetary utility functions. Pisa lecture notes. http://www. math.ethz.ch/ delbaen.

23. Delbaen F., Schachermayer $W$. A general version of the fundamental theorem of asset pricing. - Math. Ann., 1994, v. 300, № 3, p. 463-520.

24. Delbaen $F$., Schachermayer $W$. The fundamental theorem of asset pricing for unbounded stochastic processes. - Math. Ann., 1998, v. 312, № 2, p. 215-250.

25. Dempster M. A. H., Evstigneev I. V., Taksar M. I. Asset pricing and hedging in financial markets with transaction costs: an approach based on the von Neumann-Gale model. - Ann. Finance, 2006, v. 2, p. 327-355.

26. Denault $M$. Coherent allocation of risk capital. - J. Risk, 2001, v. 4, № 1, p. 1-34.

27. Detlefsen K., Scandolo G. Conditional and dynamic convex risk measures. - Finance Stoch., 2005, v. 9, № 4, p. 539-561.

28. Dowd K. Spectral risk measures. - Financial Engineering News, 2005, v. 42, p. 11-12.

29. Duffie D., Richardson H. R. Mean-variance hedging in continuous time. - Ann. Appl. Probab., 1991, v. 1, № 1, p. 1-15.

30. El Karoui $N$. Les aspects probabilistes du contrôle stochastique. - Lecture Notes in Math., 1981, v. 876, p. 73-238.

31. Fischer T. Risk capital allocation by coherent risk measures based on one-sided moments. - Insurance Math. Econom., 2003, v. 32, № 1, p. 135-146.

32. Floret K. Weakly compact sets. - Lecture Notes in Math., 1980, v. 801, p. 1-123.

33. Föllmer H., Leukert P. Quantile hedging. - Finance Stoch., 1999, v. 3, № 3, p. 251273.

34. Föllmer H., Schied A. Convex measures of risk and trading constraints. - Finance Stoch., 2002, v. 6, № 4 p. 429-447.

35. Föllmer H., Schied A. Robust preferences and convex measures of risk. Advances in Finance Stoch., Essays in Honor of Dieter Sondermann. Ed. by K. Sandmann, P. Schönbucher. Berlin: Springer, 2002, p. 39-56.

36. Föllmer H., Schied A. Stochastic Finance. An Introduction in Discrete Time. Berlin: de Gruyter, 2004, 459 p.

37. Harrison J. M., Kreps D. M. Martingales and arbitrage in multiperiod securities markets. - J. Economic Theory, 1979, v. 20, p. 381-408.

38. Harrison J.M., Pliska S.R. Martingales and stochastic integrals in the theory of continuous trading. - Stochastic Process. Appl., 1981, v. 11, № 3, p. 215-260.

39. Jacod J., Shiryaev A.N. Local martingales and the fundamental asset pricing theorems in the discrete-time case. - Finance Stoch., 1998, v. 2, № 3, p. 259-273.

40. Jaschke S., Küchler U. Coherent risk measures and good deal bounds. - Finance Stoch., 2001, v. 5, № 2, p. 181-200.

41. Jobert A., Rogers L. C. G. Pricing operators and dynamic convex risk measures. Preprint. Cambridge: Cambridge Univ., 2005;

http://www.statslab.cam.ac.uk/ chris; www.gloriamundi.org.

42. Jouini E., Kallal $H$. Martingales and arbitrage in securities markets with transaction costs. - J. Economic Theory, 1995, v. 66, № 1, p. 178-197.

43. Jouini E., Meddeb M., Touzi N. Vector-valued coherent risk measures. - Finance Stoch., 2004, v. 8, № 4, p. 531-552.

44. Кабанов Ю.М., Крамков Д.О. Отсутствие арбитража и эквивалентные мартингальные меры: новое доказательство теоремы Харрисона-Плиски. - Теория вероятн. и ее примен., 1994 , v. 39, в. 3, р. 523-527. 
45. Kabanov Yu. M., Rásonyi M., Stricker C. No-arbitrage criteria for financial markets with efficient friction. - Finance Stoch., 2002, v. 6, № 3, p. 371-382.

46. Kabanov Yu.M., Stricker C. A teacher's note on no-arbitrage criteria. - Lecture Notes in Math., 2001, v. 1755, p. 149-152.

47. Kabanov Yu.M., Stricker C. The Harrison-Pliska arbitrage pricing theorem under transaction costs. - J. Math. Econom., 2001, v. 35, № 2, p. 185-196.

48. Kalkbrenner M. An axiomatic approach to capital allocation. - Math. Finance, 2005, v. 15 , № 3, p. 425-437.

49. Kusuoka $S$. On law invariant coherent risk measures. - Adv. Math. Econom., 2001, v. 3, p. 83-95.

50. Larsen K., Pirvu T., Shreve S., Tütüncü R. Satisfying convex risk limits by trading. Finance Stoch., 2005, v. 9, № 2, p. 177-195.

51. Leventhal S., Skorokhod A.V. On the possibility of hedging options in the presence of transaction costs. - Ann. Appl. Probab., 1997, v. 7, № 2, p. 410-443.

52. Marrison C. The Fundamentals of Risk Measurement. New York: McGraw Hill, 2002.

53. Мельников А.В., Нечаев М.Л. К вопросу о хеджировании платежных обязательств в среднеквадратическом. - Теория вероятн. и ее примен., 1998, т. 43, B. 3 , c. $672-691$.

54. Overbeck L. Allocation of economic capital in loan portfolios. - Lecture Notes in Statist., 1999, v. 147, p. 1-17.

55. Riedel F. Dynamic coherent risk measures. - Stochastic Process. Appl., 2004, v. 112, № 2, p. 185-200.

56. Rogers L.C. G. Equivalent martingale measures and no-arbitrage. - Stochastics Stochastics Rep., 1994, v. 51, № 1-2, p. 41-49.

57. Roorda B., Schumacher J. M., Engwerda J. Coherent acceptability measures in multiperiod models. - Math. Finance, 2005, v. 15, № 4, p. 589-612.

58. Schachermayer $W$. A Hilbert space proof of the fundamental theorem of asset pricing in finite discrete time. - Insurance Math. Econom., 1992, v. 11, № 4, p. 249-257.

59. Schachermayer $W$. The fundamental theorem of asset pricing under proportional transaction costs in finite discrete time. - Math. Finance, 2004, v. 14, № 1, p. 19-48.

60. Schied A. Risk measures and robust optimization problems. - Stoch. Models, 2006, v. 22 , № 4, p. $753-831$.

61. Schweizer M. Variance-optimal hedging in discrete time. - Math. Oper. Res., 1995, v. 20 , № 1 , p. $1-32$.

62. Schweizer M. A guided tour through quadratic hedging approaches. Option Pricing, Interest Rates, and Risk Management. Ed. by E. Jouini, M. Musiela, and J. Cvitanic. Cambridge: Cambridge, Univ. Press, 2001, p. 538-574.

63. Sekine J. Dynamic minimization of worst conditional expectation of shortfall. Math. Finance, 2004, v. 14, № 4, p. 605-618.

64. Ширяев А. Н. Основы стохастической финансовой математики, т. 1, 2. М.: Фазис, 2004, $1017 \mathrm{c}$.

65. Soner H. M., Shreve S. E., Cvitanić J. There is no nontrivial hedging portfolio for option pricing with transaction costs. - Ann. Appl. Probab., 1995, v. 5, № 2, p. 327-355.

66. Staum J. Fundamental theorems of asset pricing for good deal bounds. - Math. Finance, 2004, v. 14, № 2, p. 141-161.

67. Stricker C. Arbitrage et lois de martingale. - Ann. Inst. H. Poincaré, 1990, v. 26, № 3, p. 451-460.

68. Szegö G. On the (non)-acceptance of innovations. - Risk Measures for the 21st Century. Ed. by G. Szegö. New York: Wiley, 2004, p. 1-9.

69. Tasche D. Expected shortfall and beyond. - J. Banking and Finance, 2002, v. 26, p. 1519-1533.

70. Yan J.A. A new look at the fundamental theorem of asset pricing. - J. Korean Math. Society, 1998, v. 35, № 3, p. 659-673. 